\title{
SPECIFYING THE AUSLANDER TRANSPOSE IN SUBMODULE CATEGORY AND ITS APPLICATIONS
}

\author{
ABDOLNASER BAHLEKEH, ALI MAHIN FALLAH AND SHOKROLLAH SALARIAN
}

\begin{abstract}
Let $(R, \mathfrak{m})$ be a $d$-dimensional commutative noetherian local ring. Let $\mathcal{M}$ denote the morphism category of finitely generated $R$-modules and let $\mathcal{S}$ be the submodule category of $\mathcal{M}$. In this paper, we specify the Auslander transpose in submodule category $\mathcal{S}$. It will turn out that the Auslander transpose in this category can be described explicitly within $\bmod R$, the category of finitely generated $R$-modules. This result is exploited to study the linkage theory as well as the Auslander-Reiten theory in $\mathcal{S}$. Indeed, a characterization of horizontally linked morphisms in terms of module category is given. In addition, motivated by a result of Ringel and Schmidmeier, we show that the Auslander-Reiten translations in the subcategories $\mathcal{H}$ and $\mathcal{G}$, consisting of all morphisms which are maximal Cohen-Macaulay $R$-modules and Gorenstein projective morphisms, respectively, may be computed within $\bmod R$ via $\mathcal{G}$-covers. Corresponding result for subcategory of epimorphisms in $\mathcal{H}$ is also obtained.
\end{abstract}

\section{Contents}

1. Introduction

2. Preliminaries

3. Auslander transpose in submodule category 7

4. Characterization of horizontally linked morphisms 12

5. Auslander-Reiten translations 14

References

\section{INTRODUCTION}

Throughout this paper $(R, \mathfrak{m})$ is a $d$-dimensional commutative noetherian local ring and $\bmod R$ is the category of all finitely generated $R$-modules. Denote by $\mathcal{M}$ the morphism category of $R$ whose objects are all $R$-homomorphisms $\mathbf{f}: A \rightarrow B$ in $\bmod R$ and morphisms are given by commutative squares. We also let $\mathcal{S}$ denote the full subcategory of $\mathcal{M}$ consisting of all $R$-monomorphisms, which is known as submodule category. It is evident that $\mathcal{M}$ is an abelian category and $\mathcal{S}$ is an additive subcategory of $\mathcal{M}$ which is closed under extensions, thus it becomes an exact category in the sense of Quillen; see [17, Appendix A].

The study of submodule categories goes back to G. Birkhoff [9], in which he has initiated to classify the indecomposable objects of the submodule category of $\mathcal{S}\left(\mathbb{Z} /<\mathbf{p}^{\mathbf{t}}>\right)$.

2000 Mathematics Subject Classification. 13H10, 16G30, 13D02, $13 \mathrm{D} 07$.

Key words and phrases. Auslander transpose; morphism category of modules; horizontally linked morphisms; Auslander-Reiten translation; almost split sequence. 
Since then there has been another approach to classify submodule categories of representation type; see $[21,23,24]$. Over noetherian algebras, many people have written beautiful papers, providing deep insights in classifying the finite Cohen-Macaulay typeness of the submodule category $\mathcal{M}$; see for instance [8], [10] and [22]. Furthermore, there are many attempts to obtain descriptions for projective, injectivi, Gorenestin projective and injective objects of $\mathcal{M}$ with respect to objects and morphisms in $\bmod R$; see e.g. $[13,15]$

One of the most powerful tools in liasion theory and representation theory, especially the Auslander-Reiten theory, is the Auslander transpose. Assume for a moment that $R$ is a (non-commutative) noetherian semiperfect ring. Assume that $M$ is a finitely generated (right) $R$-module and $P_{1} \stackrel{f}{\longrightarrow} P_{0} \longrightarrow M \longrightarrow 0$ a minimal projective presentation of $M$. Dualizing this sequence by $(-)^{*}=\operatorname{Hom}_{R}(-, R)$, the Auslander transpose of $M$, denoted by $\operatorname{Tr}_{R} M$, is defined as $\operatorname{Cok}\left(f^{*}\right)$.

It is well-understood that submodule categories are much more complicated than the underlying module category $\bmod R$. So giving a description of the Auslander transpose of objects of submodule category $\mathcal{S}$ in terms of module category, seems to be of much interest. Inspired by this idea, in this manuscript we intend to specify the Auslander transpose for objects in the submodule category $\mathcal{S}$. In this direction, after showing that any object of $\mathcal{S}$ admits a projective cover, we compute the Auslander transpose of a given object $(A \stackrel{\mathbf{f}}{\rightarrow} B) \in \mathcal{S}, \operatorname{Tr}_{\mathcal{M}} \mathbf{f}$, by applying the dual functor $\operatorname{Hom}_{\mathcal{M}}(-, \mathbf{r})$ to the minimal projective presentation of $\mathbf{f}$, in which $\mathbf{r}$ denotes the split monomorphism $(R \rightarrow R \oplus R)$. It will turn out that the Auslander transpose in the morphism category can be computed directly within the category of $\bmod R$. Precisely, it is shown that for a given object $(A \stackrel{\mathbf{f}}{\rightarrow} B) \in \mathcal{S}$, there is a projective $R$-module $Q$ such that $\operatorname{Tr}_{\mathcal{M}} \mathbf{f}=$ $\left(\operatorname{Tr}_{R}\right.$ Cokf $\left.\rightarrow \operatorname{Tr}_{R} B \oplus Q\right)$; see Proposition 3.4. This technique is exploited to investigate the linkage theory in submodule category $\mathcal{S}$. The theory of linkage of algebraic varieties was introduced by Peskine and Szpiro in [25] and generalized to module theoretic version by Martsinkovsky and Strooker in [19]. Indeed, they generalized the theory for wild class of rings, including non-commutative semiperfect noetherian rings $\Lambda$ by using the composition of two functors: transpose and syzygy. A finitely generated $\Lambda$-module $M$ and a $\Lambda^{\mathrm{op}}$-module $N$ are said to be horizontally linked, if $M \cong \lambda N$ and $N \cong \lambda M$ in which $\lambda=\Omega \operatorname{Tr}$. Equivalently, $M$ is horizontally linked (to $\lambda M$ ) if and only if $M \cong \lambda^{2} M$; see $[19$, Definition 2]. Following this, we say that an object $(A \stackrel{\mathbf{f}}{\rightarrow} B) \in \mathcal{S}$ is horizontally linked, if $\mathbf{f} \cong \lambda^{2} \mathbf{f}$, whenever $\lambda=\Omega_{\mathcal{M}}^{1} \operatorname{Tr}_{\mathcal{M}}$. Surprisingly, it will be observed that an object $(A \stackrel{\mathbf{f}}{\rightarrow} B) \in \mathcal{S}$ horizontally linked if and only if $A$ and $B$ are horizontally linked $R$ modules; see Theorem 4.6. As an application, we deduce that any Gorenstein projective objects of $\mathcal{M}$, is horizontally linked.

A nice result of Ringel and Schmidmeier indicates that, over an artin algebra $\Lambda$, the Auslaner-Reiten translation is the submodule category can be computed within mod $\Lambda$ by using their construction of minimal monomorphisms; see [21, Theorem 5.1]. It is quite natural to ask whether there is an analogues of Ringel and Schmidmeier's result for noetherian algebra or not. Motivated by such a question, in the paper's final section we will apply the description of the Auslander transpose in submodule category obtained in Sec. 3 and answer the raised question affirmatively. To be precise more, assume that $Q: \bullet \rightarrow \bullet$ is a quiver and $R$ is a complete Gorenstein ring. Since the morphism category 
$\mathcal{M}$ is equivalent to the category of finitely generated modules over the path algebra $R \mathcal{Q}$, $\bmod R \mathcal{Q}$, the main result of Auslander and Reiten in [6] asserts that the full subcategory of $\mathcal{M}$ whose objects are maximal Cohen-Macaulay $R$-modules, denoted by $\mathcal{H}$, admits almost split sequences. In particular, for a given non-projective indecomposable object f of $\mathcal{H}$ which is locally projective on the punctured spectrum of $R$, in the sense we will define later, the Auslander-Reiten translation of $\mathbf{f}$ in $\mathcal{H}, \tau_{\mathcal{H}}(\mathbf{f})$, has the form $\left(\Omega_{\mathcal{H}}^{d} \operatorname{Tr}_{\mathcal{H}}(\mathbf{f})\right)^{\prime}$, where $(-)^{\prime}=\operatorname{Hom}_{R}(-, R)$. On the other hand, with the aid of an interesting result due to Liu, Ng and Paquette [18, Proposition 4.5], we have been able to show that the subcategories $\mathcal{G}$ and $\mathcal{E}$ of $\mathcal{H}$ admit almost split sequences, whenever $\mathcal{G}$ and $\mathcal{E}$ denote respectively the full subcategories of $\mathcal{H}$ consisting of Gorenstein projective objects and epimorphisms. Moreover, it will turn out that the Auslander-Reiten translations $\tau_{\mathcal{H}}$, $\tau_{\mathcal{G}}$ and $\tau_{\mathcal{E}}$ can be computed directly in $\bmod R$ by using $\mathcal{G}$-cover and $\mathcal{E}$-envelope of $\tau_{R}$ and $\tau_{R}^{-1}$. More specifically, let $\mathbf{f}$ (resp. $\mathbf{g}$ ) be an indecomposable non-projective (resp. non-injective) object of $\mathcal{G}$ (resp. $\mathcal{E}$ ) with is locally projective on the punctured spectrum of $R$, then we have the following;

(1) $\tau_{\mathcal{H}}(\mathbf{f}) \cong \mathbf{C o k}\left(\operatorname{red}\left(\mathcal{G}-\operatorname{cov} \tau_{R} \mathbf{f}\right)\right)$ $\cong \operatorname{red}\left(\mathcal{E}-e n v \tau_{R} \operatorname{Cok}(\mathbf{f})\right)$.

(2) $\tau_{\mathcal{G}}(\mathbf{f}) \cong \operatorname{red}\left(\mathcal{G}-\operatorname{cov} \tau_{R} \operatorname{Cok}(\mathbf{f})\right)$.

(3) $\tau_{\mathcal{E}}(\mathbf{g}) \cong \operatorname{Cok}\left(\operatorname{red}\left(\mathcal{G}-\operatorname{cov} \tau_{R} \mathbf{g}\right)\right)$.

Here, $\operatorname{Cok}(A \stackrel{\mathbf{f}}{\rightarrow} B)$ denotes the natural projection $B \rightarrow \operatorname{Cok}(\mathbf{f})$. The corresponding results for $\tau^{-1}$ are also obtained.

\section{Preliminaries}

In this section, we recall basic definitions and fundamental facts for later use. Let us begin by recalling a characterization of projective and injective objects in the morphism category $\mathcal{M}$.

2.1. It is known that for a ring $R$, the projective objects of $\mathcal{M}$ are direct sums of the elements of the form $P \stackrel{1}{\rightarrow} P$ and $0 \rightarrow P$, where $P$ runs among all finitely generated projective $R$-modules, whereas every injective object in $\mathcal{M}$ is a product of the elements of the form $I \stackrel{1}{\rightarrow} I$ and $I \rightarrow 0$ in which $I$ runs among all injective $R$-modules. More precisely, each indecomposable projective object of $\mathcal{M}$ is one of the mentioned form, whenever $P$ is an indecomposable projective $R$-module; see [21, Lemma 1.3]. Actually, the category $\mathcal{M}$ is clearly equivalent to the category of modules over the path algebra $R \mathcal{Q}$ of the quiver $\mathcal{Q}: \bullet \rightarrow \bullet$ over $R$; see [7]. Hence, among other sources, the statements about projective and injective objects in $\mathcal{M}$ are just special cases of the main results in [12] and [13], respectively. We denote by $\operatorname{proj} \mathcal{M}$ and $\operatorname{inj} \mathcal{M}$ the class of all projective objects and injective objects of $\mathcal{M}$, respectively. Note that since $R$ is a commutative noetherian ring, $\mathcal{M}$ may be considered as a noetherian $R$-algebra in the sense of [2].

2.2. Convention. Throughout the paper we assume:

(1) $R$ is a $d$-dimensional commutative noetherian local ring with maximal ideal $\mathfrak{m}$ and $\Lambda$ is a noetherian $R$-algebra, that is, an $R$-algebra which is finitely generated as an $R$-module. Unless specified explicitly, all modules are assumed to be finitely generated right modules. 
(2) For a given $R$-module $M$ any non-negative integer $n, \Omega^{n} M$, stands for the $n$th syzygy of a minimal projective resolution of $M$, and so it is uniquely determined, up to isomorphism.

(3) For a given object $(A \stackrel{\mathbf{f}}{\rightarrow} B) \in \mathcal{M}$, we let $\operatorname{Ker}(\mathbf{f})$ denote the inclusion map $\operatorname{Ker}(\mathbf{f}) \rightarrow$ $A$, whereas $\operatorname{Cok}(\mathbf{f})$ stands for natural projection $B \rightarrow \operatorname{Cok}(\mathbf{f})$, as objects of $\mathcal{M}$.

2.3. Gorenstein projective modules. A finitely generated $\Lambda$-module $M$ is said to be in $G(\Lambda)$, which is also called Gorenstein projective, if it is a syzygy of an acyclic complex of projective $\Lambda$-modules; $\cdots \longrightarrow P_{n+1} \stackrel{\delta_{n+1}}{\longrightarrow} P_{n} \stackrel{\delta_{n}}{\longrightarrow} P_{n-1} \longrightarrow \cdots$, which remains acyclic after applying the functor $\operatorname{Hom}_{\Lambda}(-, P)$ for any projective $\Lambda$-module $P$. It is worth pointing out that over commutative Gorenstein local rings, the class of all maximal Cohen-Macaulay modules and Gorenstein projective modules are the same.

Lemma 2.4. Let $R$ be a complete local ring and $\Lambda$ a noetherian $R$-algebra. Let $A$ be an indecomposable non-projective and Gorenstein projective $\Lambda$-module. Then the same is true for $\Omega^{i} A$, for all $i \geq 0$.

Proof. If $i=0$, there is nothing to prove. In order to prove the claim for $i>0$, it suffices to consider only the case $i=1$. Since $R$ is complete, by invoking [11, pp. 132] one deduces that $\Lambda$ is semiperfect, meaning that every $\Lambda$-module admits a projective cover. Taking a short exact sequence of $\Lambda$-modules; $0 \rightarrow \Omega^{1} A \rightarrow P \rightarrow A \rightarrow 0$, whenever $P \rightarrow A$ is a projective cover of $A$, we claim that $\Omega^{1} A$ is stable, that is, it has no any projective direct summand. Assume on the contrary that $\Omega^{1} A$ is written as $X \oplus Q$, for some non-zero projective $\Lambda$-module $Q$ and so, one has a short exact sequence $0 \rightarrow$ $X \oplus Q \rightarrow P \rightarrow A \rightarrow 0$. Hence, due to the fact that $\operatorname{Ext}_{\Lambda}^{1}(A, Q)=0$, one may infer that the morphism $Q \rightarrow P$ is split, which contradicts with the fact that $P \rightarrow A$ is a projective cover of $A$. On the other hand, according to [20, Exercise 6], $\bmod \Lambda$ has KrullSchmidt property. Hence, $A$ being indecomposable yields that the endomorphism ring of $A, \operatorname{End}_{\Lambda}(A)$, is local and so is $\operatorname{End}_{\Lambda}(A)$. Since $\operatorname{Ext}_{\Lambda}^{1}(A, P)=0$, for all projective $\Lambda$ modules $P$, one may apply a standard argument to show that $\underline{\operatorname{End}}_{\Lambda}(A) \cong \underline{\operatorname{End}}_{\Lambda}\left(\Omega^{1} A\right)$ implying the latter is a local ring. As $\Omega^{1} A$ is stable, Proposition 2.5 of [3] enforces that $\mathfrak{P}\left(\Omega^{1} A, \Omega^{1} A\right)$ is contained in $J\left(\operatorname{End}_{\Lambda}\left(\Omega^{1} A\right)\right)$, where $\mathfrak{P}\left(\Omega^{1} A, \Omega^{1} A\right)$ consists of all endomorphisms of $\Omega^{1} A$ which factor through projective $\Lambda$-modules. This, in particular, gives that $\operatorname{End}_{\Lambda}\left(\Omega^{1} A\right)$ is a local ring ensuring that $\Omega^{1} A$ is indecomposable. The proof then is complete.

2.5. It should be observed that the definition of Gorenstein projective object may be generalized to the morphism category. In fact, a given object $(A \stackrel{\mathbf{f}}{\rightarrow} B) \in \mathcal{M}$ is Gorenstein projective if and only if $\mathbf{f} \in \mathcal{S}$ and $A, \operatorname{Cok} \mathbf{f} \in \mathrm{G}(R)$; see $[13,15]$. The full subcategory of $\mathcal{M}$ consisting of Gorenstein projective objects will be denoted by $\mathcal{G}$. Let $\mathcal{H}$ stand for the full subcategory of $\mathcal{M}$ consisting of all objects $(A \stackrel{\mathbf{f}}{\rightarrow} B)$ in which $A, B \in \mathrm{G}(R)$. So, $\mathcal{G}$ becomes a full subcategory of $\mathcal{H}$ as well. Also, the full subcategory of $\mathcal{H}$ which consists all epimorphisms will be denoted by $\mathcal{E}$. Evidently, $\mathcal{G}$ and $\mathcal{E}$ are extension closed and hence they become as exact categories. It can be easily verified that every indecomposable injective object of $\mathcal{H}$ is of the form $P \rightarrow 0$ and $P \stackrel{1}{\rightarrow} P$, where $P$ is an indecomposable projective $R$-module. In particular, one has the equalities proj $\mathcal{G}=\operatorname{inj} \mathcal{G}=\operatorname{proj} \mathcal{H}$. Moreover, the equalities $\operatorname{proj} \mathcal{M}=\operatorname{proj} \mathcal{S}=\operatorname{proj} \mathcal{H}=\operatorname{proj} \mathcal{G}$ hold trivially. Since the functor $(-)^{\prime}=\operatorname{Hom}_{R}(-, R): \mathrm{G}(R) \rightarrow \mathrm{G}(R)$ is a duality, clearly it induces a duality 
between the full subcategories $\mathcal{G}$ and $\mathcal{E}$ of $\mathcal{H}$ implying the equalities projE $\mathcal{E}=\operatorname{inj} \mathcal{E}=\operatorname{inj} \mathcal{H}$, meaning that $\mathcal{E}$ is a Frobenius category.

2.6. (Pre) Coves And (PRE) Envelopes. Let $\mathcal{A}$ be an exact category. A morphism $\mathrm{f}: X \rightarrow Y$ in $\mathcal{A}$ is right minimal if any endomorphism $\mathbf{g}: X \rightarrow X$ satisfying $\mathbf{f g}=\mathbf{f}$ is an automorphism; and left minimal if any endomorphism $\mathbf{h}: Y \rightarrow Y$ such that $\mathbf{h f}=\mathbf{f}$ is an automorphism. Let $\mathcal{C}$ be a full subcategory of $\mathcal{A}$ and let $X$ be an object in $\mathcal{A}$. A morphism $\mathbf{f}: M \rightarrow X$ with $M \in \mathcal{C}$ is called a $\mathcal{C}$-precover of $X$, if $\operatorname{Hom}_{\mathcal{A}}(1, \mathbf{f})$ : $\operatorname{Hom}_{\mathcal{A}}(L, M) \longrightarrow \operatorname{Hom}_{\mathcal{A}}(L, X)$ is surjective for any $L \in \mathcal{C}$; and a $\mathcal{C}$-cover if, in addition, f is right minimal. In this case we may write $M=\mathcal{C}$-cov $X$. Dually, we say that a morphism $\mathbf{h}: X \rightarrow N$ with $N \in \mathcal{C}$ is a $\mathcal{C}$-preenvelope if $\operatorname{Hom}_{\mathcal{A}}(\mathbf{h}, 1): \operatorname{Hom}_{\mathcal{A}}(N, L) \rightarrow$ $\operatorname{Hom}_{\mathcal{A}}(X, L)$ is surjective for any $L \in \mathcal{C}$; and a $\mathcal{C}$-envelope if, in addition, $\mathbf{h}$ is left minimal. We call $N$ is as $\mathcal{C}$-env $X$.

2.7. Almost split sequences. Let $\mathcal{A}$ be an exact category and $\mathbf{f}: B \rightarrow C$ be a morphism in $\mathcal{A}$. $\mathbf{f}$ is said to be right almost split provided it is not a split epimorphism and every morphism $\mathbf{g}: X \rightarrow C$ which is not a split epimorphism factors through $\mathbf{f}$. A non split exact sequence $0 \rightarrow A \stackrel{\mathbf{f}}{\rightarrow} B \stackrel{\mathrm{g}}{\rightarrow} C \rightarrow 0$ is called an almost split provided the endomorphism ring of $A$ is local and $\mathbf{g}$ is right almost split. We remark that, since this sequence is unique up to isomorphism for $A$ and for $C$, we may write $A=\tau_{\mathcal{A}} C$ and $C=\tau_{\mathcal{A}}^{-1} A$; see [7]. The notion of almost split sequences was first defined by Auslander and Reiten in [5] during their study of module category of an artin algebra. It was in this setting that they gave the first existence theorem for almost split sequences.

2.8. Let $\mathbf{f}$ and $\mathbf{g}$ be arbitrary objects of $\mathcal{M}$. We say that a morphism $\mathbf{u}: \mathbf{f} \rightarrow \mathbf{g}$ is injectively trivial, provided it factors through an injective object in $\mathcal{M}$. We denote by $\overline{\mathcal{M}}$, the injectively stable category of $\mathcal{M}$, namely, the objects of $\overline{\mathcal{M}}$ are the same as those of $\mathcal{M}$, and for objects $\mathbf{f}, \mathbf{g}$ of $\mathcal{M}$, the set of morphisms from $\mathbf{f}$ to $\mathbf{g}$ is defined by $\operatorname{Hom}_{\overline{\mathcal{M}}}(\mathbf{f}, \mathbf{g})=\overline{\operatorname{Hom}}_{\mathcal{M}}(\mathbf{f}, \mathbf{g})=\operatorname{Hom}_{\mathcal{M}}(\mathbf{f}, \mathbf{g}) / \mathfrak{I}_{\mathcal{M}}(\mathbf{f}, \mathbf{g})$, whereas, $\mathfrak{I}_{\mathcal{M}}(\mathbf{f}, \mathbf{g})$ consist of all injectively trivial maps from $\mathbf{f}$ to $\mathbf{g}$. For a given $\mathbf{u} \in \operatorname{Hom}_{\mathcal{M}}(\mathbf{f}, \mathbf{g})$, we shall denote by $\overline{\mathbf{u}}$, its image in $\operatorname{Hom}_{\overline{\mathcal{M}}}(\mathbf{f}, \mathbf{g})$. Analogously, projectively trivial maps in $\mathcal{M}$ and the projectively stable category of $\mathcal{M}$, denoted by $\underline{\mathcal{M}}$, is defined. In addition, for a given noetherin $R$-algebra $\Lambda$, the projectively stable category of $\bmod \Lambda$ will be denoted by $\underline{\bmod } \Lambda$. It is known that for a given $\Lambda$-homomorphism $f: M \rightarrow N$ in $\bmod \Lambda, \Omega_{\Lambda}^{i}(f)$ : $\Omega_{\Lambda}^{i} M \rightarrow \Omega_{\Lambda}^{i} N$ is unique, up to projectively trivial maps and so, it is uniquely determined in $\underline{\bmod } \Lambda$.

Remark 2.9. Let $\mathbf{f}, \mathbf{g}: A \rightarrow B$ be objects in $\mathcal{M}$ such that $\mathbf{g}-\mathbf{f}$ factorizes through a projective $R$-module $Q$. That is, there are $R$-homomorphisms $\mathbf{e}: A \rightarrow Q$ and $\mathbf{u}: Q \rightarrow B$ such that $\mathbf{f}-\mathbf{g}=\mathbf{u e}$. The commutative diagram below in $\bmod R$;

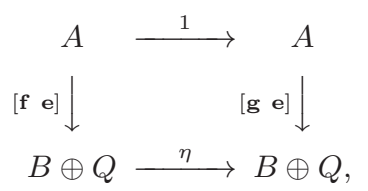

where $\eta=\left[\begin{array}{ll}1 & 0 \\ \mathbf{u} & 1\end{array}\right]$, induces that the objects $[\mathbf{f} \mathbf{e}]$ and $[\mathbf{g}$ e] are isomorphic in $\mathcal{M}$.

For a given object $\mathbf{h}$ in $\mathcal{M}$, we write $\operatorname{red}(\mathbf{h})$ for the reduced $\mathbf{h}$, i.e., the object obtained by deleting any non-trivial projective direct summands from the representations of $\mathbf{h}$ in $\mathcal{M}$. One should observe that if $\mathbf{h}$ belongs to $\mathcal{G}$ or $\mathcal{E}$, then $\operatorname{red}(\mathbf{h})$ is obtained by deleting 
any non-trivial projective or injective direct summand of $\mathbf{h}$ in $\mathcal{G}$ or $\mathcal{E}$, respectively, since these are Frobenius categories.

2.10. Let $(A \stackrel{\mathbf{f}}{\rightarrow} B) \in \mathcal{H}$ be arbitrary. Assuming $\mathbf{p}^{\prime}: P \rightarrow \operatorname{Cok}(\mathbf{f})$ as a projective cover of $\operatorname{Cok}(\mathbf{f})$, there is an $R$-homomorphism $\mathbf{p}: P \rightarrow B$ such that $\mathbf{p}^{\prime}=\mathbf{g p}$, where $\mathbf{g}: B \rightarrow$ $\operatorname{Cok}(\mathbf{f})$ is the canonical epimorphism. It is fairly easy to see that $[\mathbf{f} \mathbf{p}]^{t}: A \oplus P \rightarrow B$ is independent of the choice of $\mathbf{p}$ and $[\mathbf{f} \mathbf{p}]^{t} \in \mathcal{E}$. Moreover, analogues to the proof of Proposition 2.4 (2) of [21], one may deduce that $\mathbf{f} \rightarrow[\mathbf{f} \mathbf{p}]^{t}$ is an $\mathcal{E}$-envelope of $\mathbf{f}$.

2.11. Let $\left(A \stackrel{\mathbf{f}}{\rightarrow} A_{1}\right)$ and $\left(C \stackrel{\mathbf{h}}{\rightarrow} C_{1}\right)$ be arbitrary objects in $\mathcal{G}$ and $\mathcal{H}$, respectively. According to the fact that where $(-)^{\prime}=\operatorname{Hom}_{R}(-, R): \mathcal{G} \rightarrow \mathcal{E}$ is a duality, one may easily deduce that the morphism $\mathbf{f} \rightarrow \mathbf{h}$ is a $\mathcal{G}$-cover of $\mathbf{h}$ if and only if $\mathbf{h}^{\prime} \rightarrow \mathbf{f}^{\prime}$ is an $\mathcal{E}$-envelope of $\mathbf{h}^{\prime}$.

Lemma 2.12. Every object of $\mathcal{H}$ admits a $\mathcal{G}$-cover.

Proof. Take an arbitrary object $(A \stackrel{\mathbf{f}}{\rightarrow} B) \in \mathcal{H}$. As $\left(B^{\prime} \stackrel{\mathbf{f}^{\prime}}{\rightarrow} A^{\prime}\right) \in \mathcal{H}$, according to 2.10, $\mathbf{f}^{\prime}$ admits an $\mathcal{E}$-envelope $\mathbf{f}^{\prime} \rightarrow\left[\mathbf{f}^{\prime} \mathbf{p}\right]^{\mathbf{t}}$ in which $\mathbf{p}: P \rightarrow A^{\prime}$ with $P \rightarrow \operatorname{Cok}\left(\mathbf{f}^{\prime}\right)$ is the projective cover of $\operatorname{Cok}\left(\mathbf{f}^{\prime}\right)$. So by making use of 2.11, [f $\left.\mathbf{e}\right] \rightarrow \mathbf{f}$ will be a $\mathcal{G}$-cover of $\mathbf{f}$, in which $\mathbf{e}=\mathbf{p}^{\prime}$. One should observe that $\operatorname{Ker}(\mathbf{f})=\left(\operatorname{Cok}\left(\mathbf{f}^{\prime}\right)\right)^{\prime} \rightarrow P^{\prime}$ is a projective envelope of $\operatorname{Ker}(\mathbf{f})$ and $\mathbf{e}: A \rightarrow P^{\prime}$ is its extension.

2.13. Evaluation functor and its adjoints. Let $\mathcal{Q}: \bullet_{1} \rightarrow \bullet_{2}$ be a quiver. Associated to any vertex $i \in\{1,2\}$, there exists a functor $e^{i}: \operatorname{rep}(\mathcal{Q}, R) \rightarrow \bmod R$, called the evaluation functor, which assigns to any representation $\mathcal{X}$ of $\mathcal{Q}$ its module at vertex $i$, denoted $\mathcal{X}_{i}$. It is proved in [14] that $e^{i}$ possesses a right and also a left adjoint, which is denoted by $e_{\rho}^{i}$ and $e_{\lambda}^{i}$, respectively. We refer the reader to [1] for precise definition of these functors. Now, since the categories $\mathcal{M}$ and $\operatorname{rep}(\mathcal{Q}, R)$ are equivalent, for a given projective $R$-module $P$, the following isomorphisms hold true:

1) $\operatorname{Hom}_{\mathcal{M}}\left(e_{\lambda}^{1}(P), e_{\lambda}^{1}(R)\right) \cong \operatorname{Hom}_{R}(P, R) \cong \operatorname{Hom}_{\mathcal{M}}\left(e_{\lambda}^{2}(P), e_{\lambda}^{2}(R)\right)$.

2) $\operatorname{Hom}_{\mathcal{M}}\left(e_{\lambda}^{1}(P), e_{\lambda}^{2}(R)\right) \cong \operatorname{Hom}_{R}\left(P,\left(e_{\lambda}^{2}(R)\right)_{1}\right) \cong \operatorname{Hom}_{R}(P, 0)$.

3) $\operatorname{Hom}_{\mathcal{M}}\left(e_{\lambda}^{2}(P), e_{\lambda}^{1}(R)\right) \cong \operatorname{Hom}_{R}\left(P,\left(e_{\lambda}^{1}(R)\right)_{2}\right) \cong \operatorname{Hom}_{R}(P, R)$.

2.14. Let $\mathcal{Q}: \bullet_{1} \rightarrow \bullet_{2}$ be a quiver with relations $e_{1} \alpha=\alpha=\alpha e_{2}$. Let $(M \stackrel{\mathrm{f}}{\rightarrow} N)$ be an arbitrary object of $\mathcal{M}$. It is evident that $M \oplus N$ with the actions; $(m, n) \cdot e_{1}=(m, 0)$, $(m, n) \cdot e_{2}=(0, n)$ and $(m, n) \cdot \alpha=(0, \mathbf{f}(m))$, for all $(m, n) \in M \oplus N$, will be a right $R \mathcal{Q}$-module. So, one can find the following quasi-inverses functors;

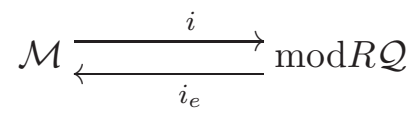

in which, $i(\mathbf{f})=M \oplus N$ for any $(M \stackrel{\mathbf{f}}{\rightarrow} N) \in \mathcal{M}$, whereas, $i_{e}(X)=X e_{1} \stackrel{\alpha}{\rightarrow} X e_{2}$ for all $X \in \bmod R \mathcal{Q}$, the category of right $R \mathcal{Q}$-modules. Inspired by this fact, we identify the objects of $\mathcal{M}$ with those in $i_{e}(\bmod R \mathcal{Q})$. Analogously, for a given object $(M \stackrel{\mathbf{f}}{\rightarrow} N) \in \mathcal{M}$, $M \oplus N$ is an $R \mathcal{Q}^{\text {op }}$-module. In particular, we have also the following quasi-inverses functors;

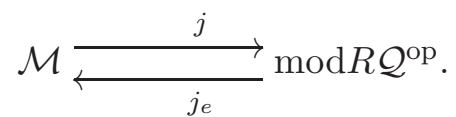


By an object in $\mathcal{M}^{\text {op }}$ we mean an object in $j_{e}\left(\bmod R \mathcal{Q}^{\text {op }}\right)$, that is, $(M \stackrel{\mathbf{f}}{\rightarrow} N)$ being in $\mathcal{M}^{\text {op }}$ means that $\mathbf{f}=\left(e_{2} X \rightarrow e_{1} X\right)$, where $X=M \oplus N$.

\section{Auslander transpose in submodule Category}

As we have mentioned in the introduction, the Auslander transpose plays a crucial role in various contexts, such as linkage theory and representation theory. Moreover, it has been long known that the submodule category is much more complicated than its underlying module theory. So specifying the Auslander transpose in submodule category within $\bmod R$ will help us in many investigations. Proposition 3.4 may be constructed in this direction. We begin with the following easy observation which is needed for our later investigation.

Lemma 3.1. Let $(A \stackrel{\mathbf{f}}{\rightarrow} B) \in \mathcal{S}$ be arbitrary. Then $\mathbf{f}$ admits a projective cover, moreover, for each $i \geq 0$, there is a projective $R$-module $Q$ such that $\Omega_{\mathcal{M}}^{i}(\mathbf{f})=\left(\Omega^{i} A \stackrel{\left[\Omega_{R}^{i} \mathbf{f} e\right]}{\longrightarrow}\right.$ $\left.\Omega^{i} B \oplus Q\right)$.

Proof. Set, for simplicity, $C=$ Cokf and $\mathbf{g}=\mathbf{C o k f}$. If $i=0$, there is nothing to prove. So assume that $i=1$. Take the following commutative diagram with exact rows and columns in $\bmod R$;

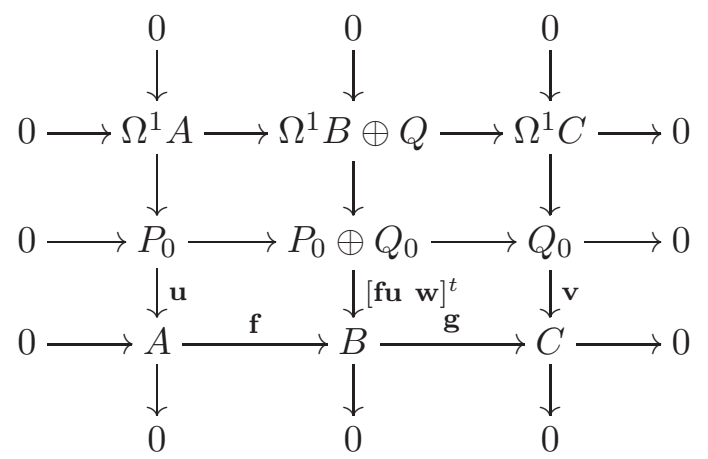

in which $\mathbf{u}: P_{0} \rightarrow A$ and $\mathbf{v}: Q_{0} \rightarrow C$ are projective covers of $R$-modules $A$ and $C$, and gw $=\mathbf{v}$. It is straightforward to see that $0 \rightarrow P_{0} \rightarrow P_{0} \oplus Q_{0} \rightarrow Q_{0} \rightarrow 0$ is a projective cover of the short exact sequence $0 \rightarrow A \stackrel{\mathrm{f}}{\rightarrow} B \stackrel{\mathrm{g}}{\longrightarrow} C \rightarrow 0$ in the category of complexes over $\bmod R$. Using this technique, one can see that $\left(P_{0} \stackrel{\left[\begin{array}{ll}1 & 0\end{array} \rightarrow\right.}{\rightarrow} P_{0} \oplus Q_{0}\right) \rightarrow(A \stackrel{\mathbf{f}}{\rightarrow} B)$ is a projective cover of $(A \stackrel{\mathbf{f}}{\rightarrow} B)$ in $\mathcal{M}$, meaning that $\Omega_{\mathcal{M}}^{1}(\mathbf{f})=\left(\Omega^{1} A \stackrel{\left[\Omega_{R}^{1} \mathbf{f} e\right]}{\longrightarrow} \Omega^{1} B \oplus Q\right)$. Hence, iterating this argument gives the desired result.

Let $\mathcal{Q}: \bullet_{1} \rightarrow \bullet_{2}$ be a quiver and $R \mathcal{Q}$ be the path algebra. According to our convention, $i_{e}(R \mathcal{Q})=\left(R e_{1} \rightarrow R e_{2} \oplus R \alpha\right)=(R \rightarrow R \oplus R)=\mathbf{r}$. We use the notation $(-)^{*}$ to denote the dual functor $\operatorname{Hom}_{\mathcal{M}}(-, \mathbf{r})$. Also, as pointed out before $(-)^{\prime}=\operatorname{Hom}_{R}(-, R)$. It can be easily checked that for any object $\mathbf{f} \in \mathcal{M}, \operatorname{Hom}_{\mathcal{M}}\left(\mathbf{f}, R e_{1} \rightarrow R e_{2} \oplus R \alpha\right)=\mathbf{f}^{*}$ is an $R \mathcal{Q}^{\mathrm{op}}$-module.

Lemma 3.2. Let $P$ be a projective $R$-module. Then with the notation and assumption of the above, the following isomorphisms hold true.

(1) $j_{e}(P \stackrel{1}{\rightarrow} P)^{*} \cong\left(0 \rightarrow P^{\prime}\right)$. 
(2) $j_{e}(0 \rightarrow P)^{*} \cong\left(P^{\prime} \stackrel{1}{\rightarrow} P^{\prime}\right)$.

Proof. We only prove the first assertion, the second one follows similarly. To this end, take the following isomorphism;

$j_{e}(P \rightarrow P)^{*} \cong e_{2} \operatorname{Hom}_{\mathcal{M}}\left(P \rightarrow P, \operatorname{Re}_{1} \rightarrow R e_{2} \oplus R \alpha\right) \longrightarrow e_{1} \operatorname{Hom}_{\mathcal{M}}\left(P \rightarrow P, R e_{1} \rightarrow \operatorname{Re}_{2} \oplus R \alpha\right)$.

On the other hand, one has the sequel isomorphism;

$e_{2} \operatorname{Hom}_{\mathcal{M}}\left(P \rightarrow P, \operatorname{Re}_{1} \rightarrow \operatorname{Re}_{2} \oplus R \alpha\right)=\operatorname{Hom}_{\mathcal{M}}\left(P \rightarrow P, 0 \rightarrow R e_{2}\right) \cong \operatorname{Hom}_{\mathcal{M}}(P \rightarrow P, 0 \rightarrow R)$,

where the right hand side is isomorphic to $\operatorname{Hom}_{\mathcal{M}}\left(e_{\lambda}^{1}(P), e_{\lambda}^{2}(R)\right)$, and so, according to 2.13 , this is equal to zero. Analogously, we have

$$
\begin{aligned}
e_{1} \operatorname{Hom}_{\mathcal{M}}\left(P \rightarrow P, R e_{1} \rightarrow R e_{2} \oplus R \alpha\right) & =\operatorname{Hom}_{\mathcal{M}}\left(P \rightarrow P, R e_{1} \rightarrow R \alpha\right) \\
& \cong \operatorname{Hom}_{\mathcal{M}}(P \rightarrow P, R \rightarrow R) \\
& \cong \operatorname{Hom}_{\mathcal{M}}\left(e_{\lambda}^{1}(P), e_{\lambda}^{1}(R)\right),
\end{aligned}
$$

in which the last one is equal to $P^{\prime}$, thanks to 2.13 . The proof then is complete.

3.3. Transpose in morphism Category. Let $(A \stackrel{\mathrm{f}}{\rightarrow} B) \in \mathcal{S}$ be given. Assume that

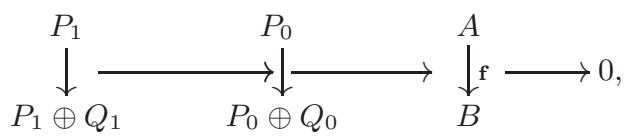

is a minimal projective presentation of $\mathbf{f}$ in $\mathcal{M}$. So, by applying the functor $(-)^{*}$ to this and using the previous lemma, one may obtain the following exact sequence in $\mathcal{M}^{\mathrm{op}}$;

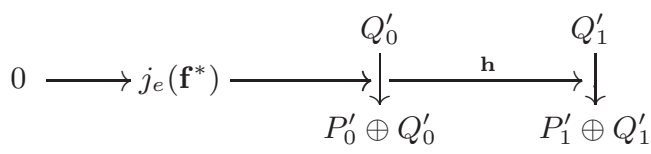

Now, the Auslander transpose of $\mathbf{f}$ in $\mathcal{M}$, denoted $\operatorname{Tr}_{\mathcal{M}}(\mathbf{f})$, is defined as $\operatorname{Cok}(\mathbf{h})$.

The result below which describes the Auslander transpose in the morphism category, plays an essential role in the remainder of the paper. We should mention the Auslander transpose in $\bmod R$ is simply denoted by $\mathrm{Tr}$.

Proposition 3.4. Let $(A \stackrel{\mathbf{f}}{\rightarrow} B) \in \mathcal{S}$ be arbitrary. Set $C=$ Cokf and $\mathbf{g}=$ Cokf. Then there exist a projective $R$-module $Q$ and $R$-homomorphisms $\mathbf{h}_{1}: \operatorname{Tr} C \rightarrow Q$ and $\mathbf{h}_{2}: Q \rightarrow \operatorname{Tr} A$ such that

(1) $\operatorname{Tr}_{\mathcal{M}}(\mathbf{f})=\left(\operatorname{Tr} C \stackrel{\left[\operatorname{Trg} \mathbf{h}_{1}\right]}{\longrightarrow} \operatorname{Tr} B \oplus Q\right)$, and the sequence $\operatorname{Tr} C \stackrel{\left[\operatorname{Trg} \mathbf{h}_{1}\right]}{\longrightarrow} \operatorname{Tr} B \oplus Q \stackrel{\left[\operatorname{Tr} \mathbf{f} \mathbf{h}_{2}\right]^{t}}{\longrightarrow}$ $\operatorname{Tr} A \rightarrow 0$ is exact.

(2) If $\operatorname{Ext}_{R}^{1}(C, R)=0$, then $\operatorname{Tr}_{\mathcal{M}}(\mathbf{f}) \in \mathcal{S}$.

Proof. (1). Take a minimal projective presentation of $(A \stackrel{\mathbf{f}}{\rightarrow} B)$ in $\mathcal{M}$ as follows;

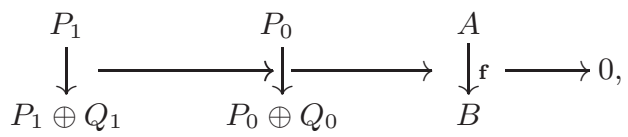

where, by Lemma 3.1, $P_{1} \rightarrow P_{0} \rightarrow A \rightarrow 0$ and $Q_{1} \rightarrow Q_{0} \rightarrow C \rightarrow 0$ are minimal projective presentations of $A$ and $C$. Applying the functor $(-)^{*}=\operatorname{Hom}_{\mathcal{M}}(-, \mathbf{r})$ to this 
sequence yields the following exact sequence in $\mathcal{M}^{\mathrm{op}}$;

$$
0 \longrightarrow j_{e}\left(\begin{array}{c}
A \\
\downarrow \\
B
\end{array}\right)^{*} \longrightarrow P_{0}^{\prime} \oplus \underset{Q_{0}^{\prime}}{\downarrow} \underset{P_{1}^{\prime} \oplus Q_{1}^{\prime}}{\downarrow} \longrightarrow \operatorname{Tr}_{\mathcal{M}}(\mathbf{f}) \longrightarrow 0 .
$$

Consequently, $\operatorname{Tr}_{\mathcal{M}}(\mathbf{f})=\left(\operatorname{Tr} C \stackrel{\left[\operatorname{Trg} \mathbf{h}_{1}\right]}{\longrightarrow} \operatorname{Tr} B \oplus Q\right)$, for some projective $R$-module $Q$. Next, consider the following commutative diagram of $R$-modules with exact rows;

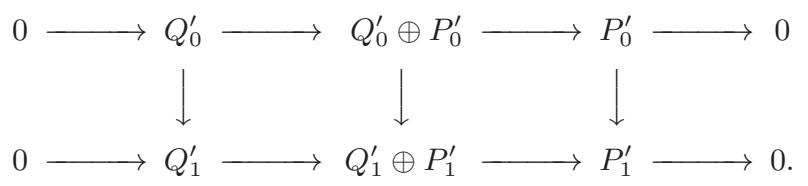

Applying Snake lemma gives us the following exact sequence;

$$
0 \rightarrow C^{\prime} \rightarrow B^{\prime} \stackrel{\mathbf{f}^{\prime}}{\rightarrow} A^{\prime} \rightarrow \operatorname{Tr} C \stackrel{\left[\operatorname{Trg} \mathbf{h}_{1}\right]}{\longrightarrow} \operatorname{Tr} B \oplus Q \stackrel{\left[\operatorname{Tr} \mathbf{f} \mathbf{h}_{2}\right]^{t}}{\longrightarrow} \operatorname{Tr} A \rightarrow 0 .
$$

So the claim follows.

(2). Since $\operatorname{Ext}_{R}^{1}(C, R)=0$, one may obtain the short exact sequence of $R$-modules; $0 \rightarrow C^{\prime} \rightarrow B^{\prime} \stackrel{\mathbf{f}^{\prime}}{\rightarrow} A^{\prime} \rightarrow 0$. So by making use of the exact sequence $(\dagger)$, one infers that the sequence $0 \rightarrow \operatorname{Tr} C \stackrel{\left[\operatorname{Trg} \mathbf{h}_{1}\right]}{\longrightarrow} \operatorname{Tr} B \oplus Q \stackrel{\left[\operatorname{Tr} \mathbf{f} \mathbf{h}_{2}\right]^{t}}{\longrightarrow} \operatorname{Tr} A \rightarrow 0$ is exact, as desired.

The following result indicates that if two objects $\mathbf{f}, \mathbf{g}: A \rightarrow B$ differ only by a map which factorizes through a projective $R$-module, then they have $\mathcal{G}$-covers simultaneously, and in particular, their $\mathcal{G}$-covers are isomorphic, up to projective summand.

Proposition 3.5. Let $\mathbf{f}, \mathbf{g}: A \rightarrow B$ be arbitrary objects of $\mathcal{M}$ such that $\mathbf{f}-\mathbf{g}$ factors through a projective $R$-module. If $\mathbf{f}$ admits a $\mathcal{G}$-cover, then so does $\mathbf{g}$ and one has the isomorphism; $\operatorname{red}(\mathcal{G}$-cov $\mathbf{f}) \cong \operatorname{red}(\mathcal{G}$-cov $\mathbf{g})$.

Proof. Take a projective $R$-module $Q$ and $R$-homomorphisms $\mathbf{e}: A \rightarrow Q$ and $\mathbf{e}^{\prime}: Q \rightarrow B$ such that $\mathbf{f}-\mathbf{g}=\mathbf{e}^{\prime} \mathbf{e}$. Consider the following short exact sequence in $\mathcal{M}$;

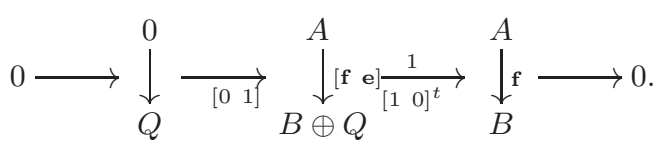

Suppose that $\mathbf{h} \rightarrow \mathbf{f}$ is a $\mathcal{G}$-cover of $\mathbf{f}$. So the following pull-back diagram

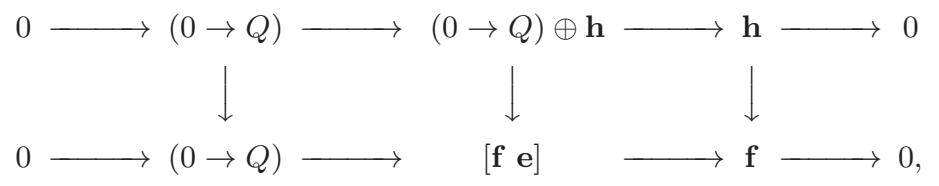

gives rise to an isomorphism $\operatorname{red}(\mathcal{G}$-cov $\mathbf{f}) \cong \operatorname{red}(\mathcal{G}$-cov $[\mathbf{f}$ e] $)$. One should note that since $(0 \rightarrow Q)$ is a projective object in $\mathcal{M}$, according to the short exact sequence $(\dagger)$, one may easily infer that $\mathbf{f}$ admits a $\mathcal{G}$-cover if and only if so does $[\mathbf{f} \mathbf{e}]$. This fact in conjunction with Remark 2.9 gives the desired result.

Remark 3.6. (1). Let $(A \stackrel{\mathrm{f}}{\rightarrow} B)$ be an object of $\mathcal{M}$ which is determined uniquely, up to projectively trivial maps. If $\mathbf{f}$ admits a $\mathcal{G}$-cover, then $\operatorname{red}(\mathcal{G}$-cov $\mathbf{f})$ is uniquely determined, up to isomorphism, thanks to Proposition 3.5.

(2). Let $\mathbf{f}, \mathbf{g}: B \rightarrow C$ be indecomposable objects in $\mathcal{E}$ such that $\mathbf{f}-\mathbf{g}$ factorizes over a 
projective $R$-module. According to the fact that $(-)^{\prime}$ is a duality on $\mathcal{H}$, one may have the isomorphism $\operatorname{red}\left(\mathcal{E}\right.$-env $\left.\Omega_{R}^{i} \mathbf{f}\right) \cong \operatorname{red}\left(\mathcal{E}\right.$-env $\left.\Omega_{R}^{i} \mathbf{g}\right)$.

From now to the end of this section, we additionally assume that $R$ is complete.

Since both functors transpose and syzygy are essential in linkage theory and representation theory, in the remainder of this section we are going to describe syzygies of transpose of certain objects in $\mathcal{M}$ via $\mathcal{G}$-covers.

3.7. We would like to add one remark here by stating the following two assertions:

(i) Assume that $(A \stackrel{\mathbf{f}}{\rightarrow} B) \in \mathcal{S}$ is given. According to Lemma 3.1, $f$ admits a minimal projective resolution in $\mathcal{M}$. So, for any $i \geq 0, \Omega_{\mathcal{M}}^{i}(\mathbf{f})$ is uniquely determined up to isomorphism. Moreover, if $\mathbf{f}$ is further assumed to be in $\mathcal{G}$, then $\Omega_{\mathcal{M}}^{i}(\mathbf{f})=\Omega_{\mathcal{H}}^{i}(\mathbf{f})$, because $\operatorname{proj} \mathcal{M}=\operatorname{proj} \mathcal{H}$.

(ii) Since $\mathcal{M}$ is equivalent to $\bmod R \mathcal{Q}$, where $\mathcal{Q}: \bullet \rightarrow \bullet$ is a quiver, by [20, Exercise 6], $\mathcal{M}$ has Krull-Schmidt property. Moreover, as $\mathcal{H}$ is a full subcategory of $\mathcal{M}$ which is closed under direct summands, $\mathcal{H}$ has Krull-Schmidt property as well.

Proposition 3.8. Let $0 \rightarrow A \stackrel{\mathbf{f}}{\rightarrow} B \stackrel{\mathrm{g}}{\rightarrow} C \rightarrow 0$ be an exact sequence of modules in $\mathrm{G}(R)$. Then the following assertions hold.

(i) $\operatorname{End}_{\mathcal{H}}(\mathbf{f})$ is a local ring if and only if $\operatorname{End}_{\mathcal{H}}(\mathrm{g})$ is so.

(ii) If $\mathbf{f}$ is indecomposable non-projective as an object of $\mathcal{H}$, then $\Omega_{\mathcal{H}}^{i}(\mathbf{f}) \cong \operatorname{red}\left(\mathcal{G}\right.$-cov $\left.\Omega_{R}^{i} \mathbf{f}\right)$ and $\operatorname{Cok}\left(\Omega_{\mathcal{H}}^{i}(\mathbf{f})\right) \cong \operatorname{red}\left(\mathcal{E}\right.$-env $\left.\Omega_{R}^{i} \mathbf{g}\right)$.

Proof. Since $(i)$ can be verified easily, we only prove part (ii). To that end, one should first note that, in view of Lemma 3.1, there is a projective $R$-module $Q$ such that $\Omega_{\mathcal{H}}^{i}(\mathbf{f})=\left(\Omega^{i} A \rightarrow \Omega^{i} B \oplus Q\right)$ and $\operatorname{Cok}\left(\Omega_{\mathcal{H}}^{i}(\mathbf{f})\right)=\left(\Omega^{i} B \oplus Q \rightarrow \Omega^{i} C\right)$ belonging to $\mathcal{G}$ and $\mathcal{E}$, respectively. According to the short exact sequence

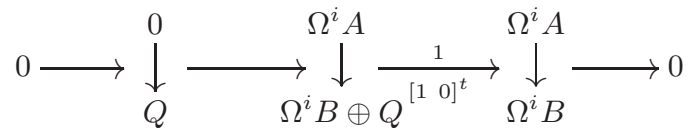

and using the fact that $(0 \rightarrow Q)$ is a projective object in $\mathcal{H}$, one deduces that the morphism

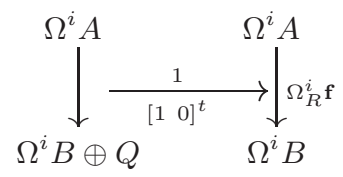

is indeed a $\mathcal{G}$-precover of $\left(\Omega^{i} A \stackrel{\Omega_{R}^{i} \mathbf{f}}{\rightarrow} \Omega^{i} B\right)$. We should stress that $\Omega_{R}^{i} \mathbf{f}$ is the map which has been appeared in $\Omega_{\mathcal{H}}^{i}(\mathbf{f})$. Moreover, by invoking Lemma 2.4 in conjunction with 3.7.(ii), one infers that $\operatorname{End}_{\mathcal{H}}\left(\Omega^{i} A \rightarrow \Omega^{i} B \oplus Q\right)$ is a local ring implying that the morphism ( $\dagger$ ) is a $\mathcal{G}$-cover of $\Omega_{R}^{i} \mathbf{f}$. Now, since $\Omega_{R}^{i} \mathbf{f}$ is unique, up to projectively trivial maps, Remark 3.6.(1) completes the proof of the first assertion. Analogously or rather dually, one may take the following short exact sequence in $\mathcal{H}$;

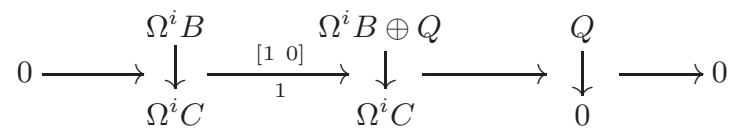


and use the fact that $(Q \rightarrow 0)$ is an injective object of $\mathcal{H}$, in order to conclude that

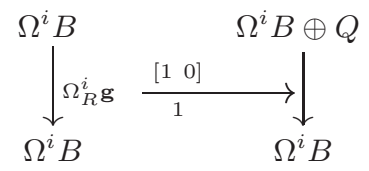

is an $\mathcal{E}$-preenvelope of $\left(\Omega^{i} B \stackrel{\Omega_{R}^{i} \mathbf{g}}{\longrightarrow} \Omega^{i} C\right)$. Furthermore, By using Lemma 2.4 again in conjunction with the first assertion, we conclude that $\operatorname{End}_{\mathcal{H}}\left(\mathbf{C o k}\left(\Omega_{\mathcal{H}}^{i}(\mathbf{f})\right)\right)$ is a local ring. This, in turn, implies that the morphism indicated by (††) is an $\mathcal{E}$-envelope of $\Omega_{R}^{i} \mathbf{g}$. So Remark 3.6.(2) completes the proof.

As an application of Proposition 3.8, we include two corollaries, which is needed in the last section of this paper.

Corollary 3.9. Let $(A \stackrel{\mathbf{f}}{\rightarrow} B)$ be an indecomposable non-projective object in $\mathcal{G}$. Then, for each $i \geq 0, \Omega_{\mathcal{H}}^{i} \operatorname{Tr}_{\mathcal{H}}(\mathbf{f})$ is isomorphic to a reduced $\mathcal{G}$-cover of $\Omega_{R}^{i} \operatorname{Tr}_{R}(\mathbf{C o k}(\mathbf{f}))$.

Proof. Set, for simplicity, $C=\operatorname{Cok}(\mathbf{f})$ and $\mathbf{g}=\operatorname{Cok}(\mathbf{f}): B \rightarrow C$. We would like to show the claim first in the case $i=0$. By Proposition 3.4, $\operatorname{Tr} \mathcal{M} \mathbf{f}=(\operatorname{Tr} C \rightarrow \operatorname{Tr} B \oplus Q)$, for some projective $R$-module $Q$ and also the sequence $0 \longrightarrow \operatorname{Tr} C \longrightarrow \operatorname{Tr} B \oplus Q \longrightarrow \operatorname{Tr} A \longrightarrow 0$ is exact. Moreover, one should note that $(A \stackrel{\mathbf{f}}{\rightarrow} B)$ belonging to $\mathcal{G}$ enforces that $\operatorname{Tr}_{\mathcal{H}}(\mathbf{f}) \cong$ $j_{e}\left(\Omega_{\mathcal{H}}^{2}(\mathbf{f})\right)^{*}$ which is indecomposable and non-projective, thanks to Lemma 2.4. In view of Proposition 3.8.(ii), $\operatorname{Cok}\left(\Omega_{\mathcal{H}}^{2}(\mathbf{f})\right)$ is isomorphic to a reduced $\mathcal{E}$-envelope of $\Omega_{R}^{2} \mathbf{g}$. In addition, it follows from the exact sequence $(\dagger)$ appeared in the proof of Proposition 3.4, that $\operatorname{Tr}_{\mathcal{H}}(\mathbf{f}) \cong j_{e}\left(\Omega_{\mathcal{H}}^{2} \mathbf{f}\right)^{*} \cong\left(\operatorname{Cok}\left(\Omega_{\mathcal{H}}^{2}(\mathbf{f})\right)^{\prime}\right.$. Consequently, $\operatorname{Tr}_{\mathcal{H}}(\mathbf{f})$ is isomorphic to a reduced $\mathcal{G}$-cover of $\left(\Omega_{R}^{2} \mathbf{g}\right)^{\prime}$, thanks to Proposition 3.8.(ii). It should be observed that, since $B$ and $C$ belong to $\mathrm{G}(R)$, one may obtain that $\left(\Omega_{R}^{2} \mathbf{g}\right)^{\prime}=(\operatorname{Tr} C \rightarrow \operatorname{Tr} B)$. So we have done. Next we are going to prove the result for $i>0$. Because of Proposition 3.8.(ii), there exists a projective $R$-module $P$ such that $\Omega_{\mathcal{H}}^{i}\left(\operatorname{Tr}_{\mathcal{H}}(\mathbf{f})\right)=\left(\Omega^{i} \operatorname{Tr} C \rightarrow \Omega^{i} \operatorname{Tr} B \oplus P\right)$ is isomorphic to a reduced $\mathcal{G}$-cover of $\Omega_{R}^{i}\left(\operatorname{Tr}_{\mathcal{H}}(\mathbf{f})\right)=\left(\Omega^{i} \operatorname{Tr} C \rightarrow \Omega^{i}(\operatorname{Tr} B \oplus Q)\right)$. Now, since $\Omega^{i}(\operatorname{Tr} B \oplus Q)=\Omega^{i} \operatorname{Tr} B$, one deduces the claim.

It is worth pointing out that for a given object $(A \stackrel{\mathbf{f}}{\rightarrow} B)$ in $\mathcal{H}$, there is an induced map $\operatorname{Tr}_{R}(\mathbf{f})=(\operatorname{Tr} B \rightarrow \operatorname{Tr} A)$ which is unique up to projectively trivial maps.

Corollary 3.10. With the notation and assumptions of the above corollary, the isomorphism $\Omega_{\mathcal{H}}^{i} \operatorname{Tr}_{\mathcal{H}}(\mathbf{f}) \cong \operatorname{Ker}\left(\operatorname{red}\left(\mathcal{E}\right.\right.$-env $\left.\left.\Omega^{i} \operatorname{Tr}_{R}(\mathbf{f})\right)\right)$ holds true.

Proof. By virtue of the second assertion of Proposition 3.4, there is a short exact sequence $0 \rightarrow \operatorname{Tr} C \rightarrow \operatorname{Tr} B \oplus Q \rightarrow \operatorname{Tr} A \rightarrow 0$, for some projective $R$-module $Q$. In particular, $\operatorname{Tr}_{\mathcal{H}}(\mathbf{f})=(\operatorname{Tr} C \rightarrow \operatorname{Tr} B \oplus Q)$ with local endomorphism ring. By applying the argument which have been used in the proof of Proposition 3.8.(ii), one may infer that $\operatorname{Tr}_{R}(\mathbf{f}) \rightarrow$ $\operatorname{Cok}\left(\operatorname{Tr}_{\mathcal{H}}(\mathbf{f})\right)$ is an $\mathcal{E}$-preenvelope of $\operatorname{Tr}_{R}(\mathbf{f})=(\operatorname{Tr} B \rightarrow \operatorname{Tr} A)$. According to Proposition 3.8.(i), we deduce that $\operatorname{End}_{\mathcal{H}}\left(\operatorname{Cok}\left(\operatorname{Tr}_{\mathcal{H}}(\mathbf{f})\right)\right)$ is a local ring ensuring $\operatorname{Cok}\left(\operatorname{Tr}_{\mathcal{H}}(\mathbf{f})\right) \cong$ $\operatorname{red}\left(\mathcal{E}\right.$-env $\left.\operatorname{Tr}_{R}(\mathbf{f})\right)$. Next suppose that $i>0$. By Proposition 3.8.(ii), $\operatorname{Cok}\left(\Omega_{\mathcal{H}}^{i} \operatorname{Tr}_{\mathcal{H}}(\mathbf{f})\right) \cong$ $\operatorname{red}\left(\mathcal{E}\right.$-env $\left.\Omega_{R}^{i} \operatorname{Tr}_{R}(\mathbf{f})\right)$ and so $\Omega_{\mathcal{H}}^{i} \operatorname{Tr}_{\mathcal{H}}(\mathbf{f}) \cong \operatorname{Ker}\left(\operatorname{red}\left(\mathcal{E}\right.\right.$-env $\left.\left.\Omega_{R}^{i} \operatorname{Tr}_{R}(\mathbf{f})\right)\right)$. Thus, the proof is complete. 


\section{Characterization of horizontally linked morphisms}

This short section deals with linkage theory in submodule category $\mathcal{S}$. Namely, inspired by the definition of horizontally linked modules, given by Martinkovsky and Strooker [19], we define horizontally linked morphisms. Moreover, results of previous section is applied to give an explicit description of horizontally linked morphisms within $\bmod R$. We begin with the following definition.

Definition 4.1. ([19, Definition 3]) Let $M$ and $N$ be finitely generated $R$-modules. $M$ and $N$ are said to be horizontally linked if $M \cong \lambda N$ and $N \cong \lambda M$, where $\lambda$ is the operator $\Omega^{1} \operatorname{Tr}_{R}$. Thus $M$ is said to be horizontally linked (to $\lambda M$ ) if and only if $M \cong \lambda^{2} M$.

Definition 4.2. Let $(A \stackrel{\mathbf{f}}{\rightarrow} B) \in \mathcal{S}$ be arbitrary. We say that $\mathbf{f}$ is horizontally linked (to $\lambda \mathbf{f}$ ) provided that $\mathbf{f} \cong \lambda^{2} \mathbf{f}$ in $\mathcal{M}$, where $\lambda=\Omega_{\mathcal{M}}^{1} \operatorname{Tr}_{\mathcal{M}}$.

Remark 4.3. Let $\mathbf{f}: A \rightarrow B$ be an arbitrary object in $\mathcal{S}$. Letting $C=\operatorname{Cok}(f)$ and $\mathbf{g}: B \rightarrow C$, we have that $\operatorname{Tr}_{\mathcal{M}} \mathbf{f}=\left(\operatorname{Tr} C \stackrel{\left[\operatorname{Trg} \mathbf{h}_{1}\right]}{\rightarrow} \operatorname{Tr} B \oplus Q\right)$, for some projective $R$-module $Q$, thanks to Proposition 3.4. Consequently, $\lambda(A \stackrel{\mathbf{f}}{\rightarrow} B)=(\lambda C \rightarrow \lambda B \oplus P)$, in which $P$ is a projective $R$-module.

Example 4.4. Let $M \in \bmod R$ be horizontally linked. Then $0 \rightarrow M$ and $M \stackrel{i d}{\rightarrow} M$, as objects of $\mathcal{S}$, are horizontally linked. Actually, by virtue of the above remark, one may obtain that $\lambda(0 \rightarrow M)=(\lambda M \rightarrow \lambda M)$ and $\lambda(M \stackrel{i d}{\rightarrow} M)=(0 \rightarrow \lambda M)$. So applying the operator $\lambda$ to theses objects again, in conjunction with the hypothesis that $M$ is horizontally linked, yields the desired result.

The next result, which gives a criterion for horizontally linkage in $\mathcal{S}$, can be proved by the same argument given in the proof of Theorem 2 of [19]. So we do not give its proof.

Proposition 4.5. $(A \stackrel{\mathbf{f}}{\rightarrow} B) \in \mathcal{S}$ is horizontally linked if and only if $\mathbf{f}$ has no projective summands and $\operatorname{Ext}_{\mathcal{M}}^{1}{ }^{\mathrm{op}}\left(\operatorname{Tr}_{\mathcal{M}}(\mathbf{f}), \mathbf{r}\right)=0$.

Now we are ready to state and prove the main result of this section. Recall that an $R$-module $M$ is said to be stable, provided it has no any non-trivial projective summand.

Theorem 4.6. Let $(A \stackrel{\mathbf{f}}{\rightarrow} B) \in \mathcal{S}$ be arbitrary such that $A$ and $B$ are stable. Then $\mathbf{f} i s$ horizontally linked if and only if so are $A$ and $B$.

Proof. Set, for simplicity, $C=$ Cokf and $\mathbf{g}=$ Cokf $: B \rightarrow C$. Let us first to show the 'sufficiency'. Since $A$ and $B$ are stable and horizontally linked, in view of [19, Theorem 2], $\operatorname{Ext}_{R}^{1}(\operatorname{Tr} A, R)=0=\operatorname{Ext}_{R}^{1}(\operatorname{Tr} B, R)$. Consider the following commutative diagram 
with exact rows and columns in $\bmod R$ :

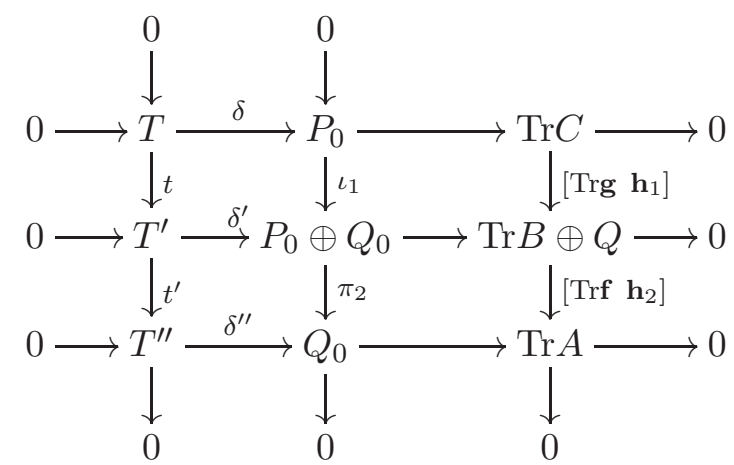

Suppose that a morphism $\left(\varphi, \varphi^{\prime}\right):\left(T \rightarrow T^{\prime}\right) \longrightarrow(R \rightarrow R \oplus R)$ in $\mathcal{M}$ is given. So, one has the following commutative diagram in $\bmod R$ :

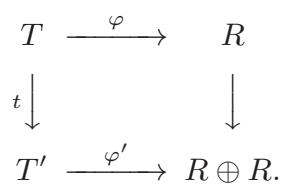

We would like to show that this morphism factors through $\left(P_{0} \rightarrow P_{o} \oplus Q_{0}\right)$. Since the split monomorphism $\mathbf{r}=(R \rightarrow R) \oplus(0 \rightarrow R)$, letting $\varphi^{\prime}=\left(\varphi_{1}^{\prime}, \varphi_{2}^{\prime}\right)$, the above commutative diagram, gives us the sequel two commutative diagrams;

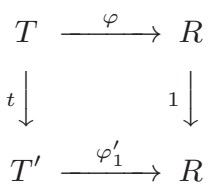

and

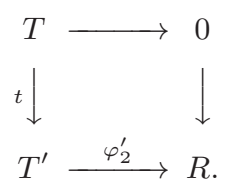

Since $\operatorname{Ext}_{R}^{1}(\operatorname{Tr} B, R)=0$, by considering the first square, we get an $R$-homomorphism $h_{2}: P_{0} \oplus Q_{0} \rightarrow R$ such that $h_{2} \delta^{\prime}=\varphi_{1}^{\prime}$. Now, assuming $h_{1}=h_{2} \iota_{1}: P_{0} \rightarrow R$, one deduces the following equalities;

$$
h_{1} \delta=h_{2} \iota_{1} \delta=h_{2} \delta^{\prime} t=\varphi_{1}^{\prime} t=\varphi .
$$

This indeed means that $\left(\varphi, \varphi_{1}^{\prime}\right)$ factors through $\left(P_{0} \rightarrow P_{0} \oplus Q_{0}\right)$. Next, it follows from the second square that $\varphi_{2}^{\prime} t=0$. So, there is an $R$-homomorphism $\varphi^{\prime \prime}: T^{\prime \prime} \rightarrow R$ such that $\varphi^{\prime \prime} t^{\prime}=\varphi_{2}^{\prime}$. On the other hand, the assumption imposed on $A$, induces an $R$ homomorphism $h^{\prime \prime}: Q_{0} \rightarrow R$ such that $\varphi^{\prime \prime}=h^{\prime \prime} \delta^{\prime \prime}$. Letting $h^{\prime}=h^{\prime \prime} \pi_{2}$, one may obtain the equalities, $h^{\prime} \delta^{\prime}=h^{\prime \prime} \pi_{2} \delta^{\prime}=h^{\prime \prime} \delta^{\prime \prime} t^{\prime}=\varphi^{\prime \prime} t^{\prime}=\varphi_{2}^{\prime}$, meaning that $\left(0, \varphi_{2}^{\prime}\right)$ factors through $\left(P_{0} \rightarrow P_{0} \oplus Q_{0}\right)$. Therefore $\operatorname{Ext}_{\mathcal{M} \text { op }}^{1}\left(\operatorname{Tr}_{\mathcal{M}}(\mathbf{f}), \mathbf{r}\right)=0$. So the claim follows from the above proposition. Next we would like to show the 'necessity'. To do this, suppose that there is an $R$-homomorphism $\varphi^{\prime \prime}: T^{\prime \prime} \rightarrow R$. So, defining the morphism $\varphi^{\prime}=\varphi^{\prime \prime} t^{\prime}: T^{\prime} \rightarrow R$, one obtains a morphism $\left(\varphi^{\prime}, \varphi^{\prime \prime}\right):\left(T^{\prime} \rightarrow T^{\prime \prime}\right) \longrightarrow(R \rightarrow R)$ in $\mathcal{M}$. So, one may obtain a morphism $\left(0, \varphi^{\prime}\right):\left(T \rightarrow T^{\prime}\right) \longrightarrow(0 \rightarrow R)$ in $\mathcal{M}$. According to the hypothesis, this map factors through $\left(P_{0} \rightarrow P_{0} \oplus Q_{0}\right)$ and in particular, we have the following commutative 
square;

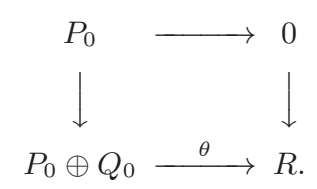

implying that any element of $P_{0}$ goes to zero under $\theta$. Using this fact, it is fairly easy to see that $\varphi^{\prime \prime}$ factors through $Q_{0}$. This indeed means that $A$ is horizontally linked, because of [19, Theorem 2]. The case for $B$ can be obtained similarly. The proof then is complete.

The next result, which is an immediate consequence of the above theorem, provides a large class of horizontally linked morphisms.

Corollary 4.7. Let $(A \stackrel{\mathbf{f}}{\rightarrow} B)$ be an arbitrary object of $\mathcal{G}$. If $A$ and $B$ are stable, then f is horizontally linked.

Proof. Since $(A \stackrel{\mathbf{f}}{\rightarrow} B) \in \mathcal{G}, A$ and $B$ belong to $\mathrm{G}(R)$ and so evidently, are horizontally linked. Hence the above theorem finishes the proof.

\section{Auslander-Reiten translations}

In this section we show that the subcategories $\mathcal{G}$ and $\mathcal{E}$ admit almost split sequences and also the Auslander-Reiten translations in $\mathcal{H}, \mathcal{G}$ and $\mathcal{E}$ of $\mathcal{M}$ are computed in terms of module case. Throughout this section $R$ is a $d$-dimensional commutative complete Gorenstein local ring.

Definition 5.1. An object $(A \stackrel{\mathbf{f}}{\rightarrow} B)$ in $\mathcal{H}$ is said to be locally projective on the punctured spectrum of $R$, provided $A_{\mathfrak{p}}$ and $B_{\mathfrak{p}}$ are projective $R_{\mathfrak{p}}$-modules and also, $\left(A_{\mathfrak{p}} \rightarrow B_{\mathfrak{p}}\right)$ is a split $R_{\mathfrak{p}}$-monomorphism, for every non-maximal prime ideal $\mathfrak{p}$ of $R$. Let $\mathbf{f}$ be an object of $\mathcal{G}$ or $\mathcal{E}$. Then $\mathbf{f}$ is locally projective on the punctured spectrum of $R$, whenever $A_{\mathfrak{p}}$ and $B_{\mathfrak{p}}$ are projective $R_{\mathfrak{p}}$-modules, for all non-maximal prime ideals $\mathfrak{p}$ of $R$.

It should be noted that, according to the description of projective objects in the morphism category $\mathcal{M}$ which has been appeared in 2.1 , and the equivalence $\bmod R \mathcal{Q} \cong$ $\mathcal{M}$, where $\mathcal{Q}$ is the quiver $\mathcal{Q}: \bullet \rightarrow \bullet$, one deduces that the subcategory of $\mathcal{H}$ consisting of all objects which are locally projective on the punctured spectrum of $R$ is equivalent to the subcategory of $\bmod R \mathcal{Q}$ consisting of all $R \mathcal{Q}$-modules $X$ which are maximal CohenMacaualy as $R$-modules and for any non-maximal prime ideal $\mathfrak{p}$ of $R, X_{\mathfrak{p}}$ is a projective $(R \mathcal{Q})_{\mathfrak{p}}$-module. Consequently, our notion of locally projective objects, agrees with the sense of [4] and [6].

Remark 5.2. Take an indecomposable non-projective object $(A \stackrel{\mathbf{f}}{\rightarrow} B)$ of $\mathcal{H}$ which is locally projective on the punctured spectrum of $R$. Due to the main result of [6], there is an almost split sequence in $\mathcal{H}$ ending in $(A \stackrel{\mathbf{f}}{\rightarrow} B)$. In particular, the Auslander-Reiten translation of $\mathbf{f}$ in $\mathcal{H}, \tau_{\mathcal{H}}(\mathbf{f})$ equals to $\left(\Omega^{d} \operatorname{Tr}_{\mathcal{H}}(\mathbf{f})\right)^{\prime}$. Inspired by this fact, for an arbitrary object $\mathbf{h}$ in $\mathcal{H}$, we let $\tau_{\mathcal{H}}(\mathbf{h})=\left(\Omega^{d} \operatorname{Tr}_{\mathcal{H}}(\mathbf{h})\right)^{\prime}$. Thus, we have the following.

Corollary 5.3. Let $(A \stackrel{\mathbf{f}}{\rightarrow} B)$ be an arbitrary indecomposable non-projective object of $\mathcal{G}$. Then one has the following isomorphisms;

$$
\begin{aligned}
\tau_{\mathcal{H}}(\mathbf{f}) & \cong \operatorname{Cok}\left(\operatorname{red}\left(\mathcal{G}-\operatorname{cov} \tau_{R} \mathbf{f}\right)\right) \\
& \cong \operatorname{red}\left(\mathcal{E}-\operatorname{env} \tau_{R} \operatorname{Cok}(\mathbf{f})\right) .
\end{aligned}
$$


Proof. In view of Corollary 3.10, $\Omega_{\mathcal{H}}^{d} \operatorname{Tr}_{\mathcal{H}}(\mathbf{f}) \cong \operatorname{Ker}\left(\operatorname{red}\left(\mathcal{E}-e n v\left(\Omega^{d} \operatorname{Tr} B \rightarrow \Omega^{d} \operatorname{Tr} A\right)\right)\right.$. So, applying the functor $(-)^{\prime}$ and using the fact that the functor $(-)^{\prime}$ preserves the short exact sequences in $\mathrm{G}(R)$ yield the first isomorphism. In order to achieve the second isomorphism, it suffices to apply 2.11 to the isomorphism $\Omega_{\mathcal{H}}^{d} \operatorname{Tr}_{\mathcal{H}}(\mathbf{f}) \cong \operatorname{red}\left(\mathcal{G}-\operatorname{cov}\left(\Omega_{R}^{d} \operatorname{Tr} \operatorname{Cok}(\mathbf{f}) \rightarrow\right.\right.$ $\left.\Omega^{d} \operatorname{Tr} B\right)$ ), given in Corollary 3.9. The proof then is complete.

In the sequel, we intend to investigate the existence of almost split sequences in the subcategories $\mathcal{G}$ and $\mathcal{E}$ and determine the Auslander-Reiten translations in these subcategories. For this purpose, we need to recall some notation and terminology from [18]. Let $\tilde{\mathcal{G}}$ and $\mathcal{E}$ stand for the full subcategories generated by the objects in $\mathcal{G}$ of $\overline{\mathcal{H}}$ and $\mathcal{E}$ of $\underline{\mathcal{H}}$, respectively. It should be observed that the injectively stable category $\overline{\mathcal{G}}$ of $\mathcal{G}$ is a quotient category of $\tilde{\mathcal{G}}$, while the projectively stable category $\underline{\mathcal{E}}$ of $\mathcal{E}$ is a quotient category of $\mathcal{E}$.

Definition 5.4. Let $\mathbf{h}$ be an object in $\mathcal{H}$. A morphism $\eta: \mathbf{f} \rightarrow \mathbf{h}$ in $\mathcal{H}$ with $\mathbf{f} \in \mathcal{G}$ is called an injectively stable $\mathcal{G}$-cover of $\mathbf{h}$ if $\bar{\eta}$ is a $\tilde{\mathcal{G}}$-cover of $\mathbf{h}$ in $\overline{\mathcal{H}}$ and $\mathbf{f}$ has no non-zero injective summand in $\mathcal{H}$. In this case, we will write $\mathbf{f}=\tilde{\mathcal{G}}$-covh. In a dual manner one can define the notion of projectively stable $\mathcal{E}$-envelope, which will be denoted by $\mathcal{E}-e n v \mathbf{h}$.

Proposition 5.5. Let [f e] $: X \rightarrow Y \oplus Q$ be an arbitrary object of $\mathcal{G}$ in which $Q$ is a projective $R$-module. Consider the natural map

$\theta$ :

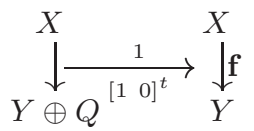

Then for any $(A \stackrel{\mathrm{g}}{\rightarrow} B) \in \mathcal{G}$, the induced map $\overline{\operatorname{Hom}}_{\mathcal{H}}\left(1_{\mathbf{g}}, \theta\right): \overline{\operatorname{Hom}}_{\mathcal{H}}(\mathbf{g},[\mathbf{f} \mathbf{e}]) \rightarrow$ $\overline{\operatorname{Hom}}_{\mathcal{H}}(\mathbf{g}, \mathbf{f})$ is an isomorphism.

Proof. In view of the short exact sequence;

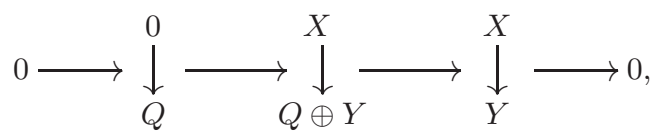

one infers that the morphism $\theta:[\mathbf{f} \mathbf{e}] \rightarrow \mathbf{f}$ is a $\mathcal{G}$-precover of $\mathbf{f}$ implying the map $\overline{\operatorname{Hom}}_{\mathcal{H}}\left(1_{\mathbf{g}}, \theta\right)$ is surjective. So, it only remains to show that $\overline{\operatorname{Hom}}_{\mathcal{H}}\left(1_{\mathbf{g}}, \theta\right)$ is an injective map. To that end, take an arbitrary object $\overline{\mathbf{h}}$ of $\overline{\operatorname{Hom}}_{\mathcal{H}}(\mathbf{g}$, [f e] $)$ such that its image is zero in $\overline{\operatorname{Hom}}_{\mathcal{H}}(\mathbf{g}, \mathbf{f})$. Therefore, $\theta \mathbf{h} \in \operatorname{Hom}_{\mathcal{H}}(\mathbf{g}, \mathbf{f})$ is injectively trivial. We show that the morphism $\mathbf{h} \in \operatorname{Hom}_{\mathcal{H}}(\mathbf{g},[\mathbf{f} \mathbf{e}])$ is injectively trivial, as well. According to 2.5, we must consider two cases. Case 1: Suppose that the image of $\mathbf{h}$ factorizes over an injective object of $\mathcal{H}$ as follows;

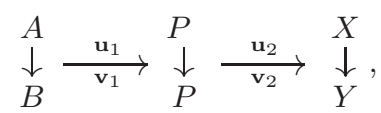

where $P$ is a projective $R$-module. Hence, we may construct the following composition map;

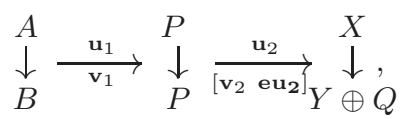


which means that $\mathbf{h}$ is an injectively trivial morphism.

Case 2. Assume that $\theta \mathbf{h} \in \operatorname{Hom}_{\mathcal{H}}(\mathbf{g}, \mathbf{f})$ factorizes over an injective object of $\mathcal{H}$ as follows;

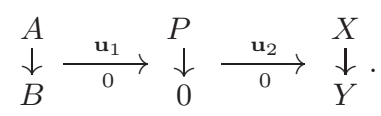

In view of the short exact sequence $0 \rightarrow A \rightarrow B \rightarrow C \rightarrow 0$ in $\mathrm{G}(R)$, one deduces that the map $\mathbf{u}_{1}: A \rightarrow P$ can be extended to the map $\mathbf{e}_{2}: B \rightarrow P$. Using this, one may construct the following composition map;

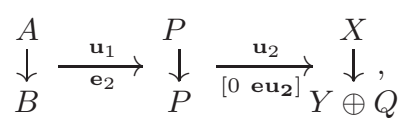

that is, $\mathbf{h}$ is injectively trivial. The proof then is completed.

The next result plays an important role in the proof the main result of this section, Theorem 5.9. The proof is fruitful from the point of view that it indicates that the injectively stable $\mathcal{G}$-cover of a given object of $\mathcal{H}$ may be recognized explicitly.

Theorem 5.6. Let $(X \stackrel{\mathbf{f}}{\rightarrow} Y)$ be an arbitrary object in $\mathcal{H}$. Then $(X \stackrel{\mathbf{f}}{\rightarrow} Y)$ admits an injectively stable $\mathcal{G}$-cover.

Proof. According to Lemma 2.12, $\left[\begin{array}{ll}1 & 0\end{array}\right]^{t}:[\mathbf{f} \mathbf{e}] \rightarrow \mathbf{f}$ is a $\mathcal{G}$-cover of $\mathbf{f}$, where $\operatorname{Ker}(\mathbf{f}) \rightarrow P$ is a projective envelope of $\operatorname{Ker}(\mathbf{f})$ and $\mathbf{e}: X \rightarrow P$ is its extension. We would like to

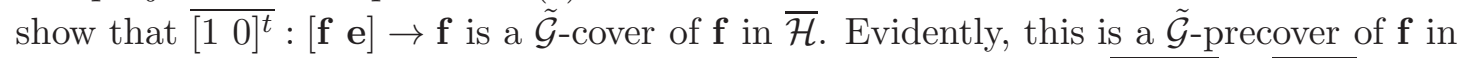

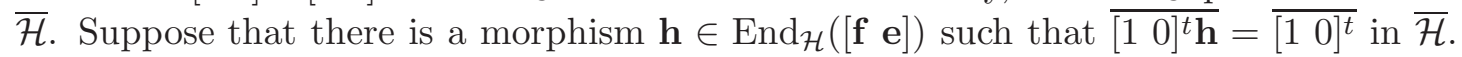
So there is an injectively trivial map $\mathbf{g} \in \operatorname{Hom}_{\mathcal{H}}\left(\left[\begin{array}{l}\mathbf{f} \\ \mathbf{e}\end{array}\right], \mathbf{f}\right)$ such that $\left[\begin{array}{ll}1 & 0\end{array}\right]^{t} \mathbf{h}-\left[\begin{array}{ll}1 & 0\end{array}\right]^{t}=\mathbf{g}$. Therefore, by the above proposition, there exists a morphism $\mathbf{h}_{\mathbf{1}} \in \operatorname{End}_{\mathcal{H}}([\mathbf{f} \mathbf{e}])$ which is injectively trivial and $\left[\begin{array}{ll}1 & 0\end{array}\right]^{t} \mathbf{h}-\left[\begin{array}{ll}1 & 0\end{array}\right]^{t}=\left[\begin{array}{ll}1 & 0\end{array}\right]^{t} \mathbf{h}_{\mathbf{1}}$. Due to the equality $\left[\begin{array}{ll}1 & 0\end{array}\right]^{t}\left(\mathbf{h}-\mathbf{h}_{\mathbf{1}}\right)=\left[\begin{array}{ll}1 & 0\end{array}\right]^{t}$ in $\mathcal{H}$ and using 2.12 again, we conclude that $\mathbf{h}-\mathbf{h}_{\mathbf{1}}$ is an isomorphism in $\mathcal{H}$ and so is $\overline{\mathbf{h}}-\overline{\mathbf{h}}_{1}$ in $\overline{\mathcal{H}}$. But, as we have seen, $\overline{\mathbf{h}}_{1}=0$ in $\overline{\mathcal{H}}$ and hence $\overline{\mathbf{h}}$ will be an isomorphism in

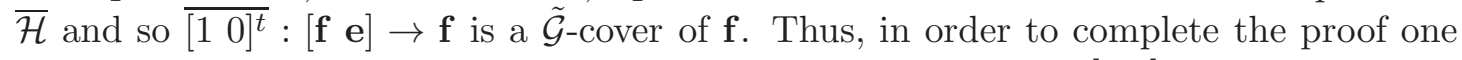
only needs to delete any non-trivial injective direct summand of [f e] in $\mathcal{H}$.

Proposition 5.7. Let $(A \stackrel{\mathbf{f}}{\rightarrow} B)$ be an arbitrary indecompasable object in $\mathcal{G}$. Then for a given object $(X \stackrel{\mathrm{g}}{\rightarrow} Y) \in \mathcal{G}$, the isomorphism $\underline{\operatorname{Hom}}_{\mathcal{H}}\left(\mathbf{g}, \tau_{R} \operatorname{Cok}(\mathbf{f})\right) \cong \underline{\operatorname{Hom}}_{\mathcal{H}}\left(\mathbf{g}, \tau_{\mathcal{H}}(\mathbf{f})\right)$ holds true.

Proof. Letting $C=\operatorname{Cok}(\mathbf{f})$, Corollary 5.3 yields that $\tau_{\mathcal{H}}(\mathbf{f})=\left(\tau_{R} B \oplus Q \rightarrow \tau_{R} C\right)$, for some projective $R$-module $Q$. Consider the following short exact sequence in $\mathcal{H}$;

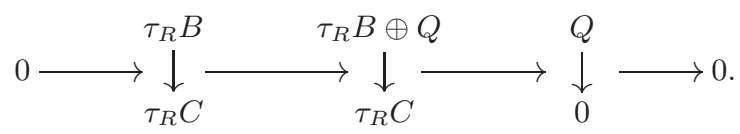

So we have an exact sequence;

$$
0 \longrightarrow \underline{\operatorname{Hom}}_{\mathcal{H}}\left(\mathbf{g}, \tau_{R} \operatorname{Cok}(\mathbf{f})\right) \longrightarrow \underline{\operatorname{Hom}}_{\mathcal{H}}\left(\mathbf{g}, \tau_{\mathcal{H}} \mathbf{f}\right) \longrightarrow \underline{\operatorname{Hom}}_{\mathcal{H}}(\mathbf{g}, Q \rightarrow 0) .
$$

Thus, in order to obtain the desired result, it suffices to show that $\underline{\operatorname{Hom}}_{\mathcal{H}}(\mathrm{g}, Q \rightarrow 0)=0$. To do this, take a morphism $\mathbf{h}=\left(\mathbf{h}_{\mathbf{1}}, 0\right) \in \operatorname{Hom}_{\mathcal{H}}(\mathbf{g}, Q \rightarrow 0)$. Since $(X \stackrel{\mathrm{g}}{\rightarrow} Y) \in \mathcal{G}$, $\mathbf{h}_{\mathbf{1}}: X \rightarrow Q$ factorizes over $\mathbf{g}$, that is, there is an $R$-homomorphism $\mathbf{h}_{\mathbf{2}}: Y \rightarrow Q$ such that $\mathbf{h}_{\mathbf{1}}=\mathbf{h}_{\mathbf{2}} \mathbf{g}$. This would imply that $\mathbf{h}$ factors through a projective object $(Q \rightarrow Q)$ meaning that $\mathbf{h}$ is a projectively trivial map. So the claim follows. 
With all these prerequisites in hand, we are now able to derive the following result.

Corollary 5.8. Let $(A \stackrel{\mathbf{f}}{\rightarrow} B)$ be an indecomposable object of $\mathcal{G}$. Then the isomorphism $\operatorname{red}\left(\mathcal{G}-\operatorname{cov} \tau_{\mathcal{H}}(\mathbf{f})\right) \cong \operatorname{red}\left(\mathcal{G}-\operatorname{cov} \tau_{R} \mathbf{C o k}(\mathbf{f})\right)$ holds true.

The theorem below, which is the main result of this section, not only guarantees the existence of almost split sequence in $\mathcal{G}$, but also computes the Auslander-Reiten translation within $\bmod R$.

Theorem 5.9. Let $(A \stackrel{\mathbf{f}}{\rightarrow} B)$ be an indecomposable non-projective object of $\mathcal{G}$ which is locally projective on the punctured spectrum of $R$. Then there is an almost split sequence in $\mathcal{G}$ ending in $\mathbf{f}$ and in particular, $\tau_{\mathcal{G}}(\mathbf{f}) \cong \operatorname{red}\left(\mathcal{G}-\operatorname{cov} \tau_{R} \mathbf{C o k}(\mathbf{f})\right)$.

Proof. According to Theorem 2.1 of [6], $\mathbf{f}$ admits an almost split sequence in $\mathcal{H}$, say $0 \longrightarrow \tau_{\mathcal{H}}(\mathbf{f}) \longrightarrow \mathbf{h} \longrightarrow \mathbf{f} \longrightarrow 0$. By virtue of Theorem 5.6, $\tau_{\mathcal{H}}(\mathbf{f})$ admits an injectively stable $\mathcal{G}$-cover, which is denoted by $\tilde{\mathcal{G}}$-cov $\tau_{\mathcal{H}}(\mathbf{f})$. In particular, the proof of Theorem 5.6 shows that $\tilde{\mathcal{G}}-\operatorname{cov} \tau_{\mathcal{H}}(\mathbf{f})=\operatorname{red}\left(\mathcal{G}-\operatorname{cov} \tau_{\mathcal{H}}(\mathbf{f})\right)$. Now, one may apply [18, Proposition 4.5] in order to conclude that $\mathbf{f}$ admits an almost split sequence in $\mathcal{G}$ and in particular, $\tau_{\mathcal{G}}(\mathbf{f})=\tilde{\mathcal{G}}-\operatorname{cov} \tau_{\mathcal{H}}(\mathbf{f})$. Hence, Corollary 5.8 finishes the proof.

Now we are going to present analogues of the above theorem for the subcategory $\mathcal{E}$. To do this, first we state the following easy observation.

Proposition 5.10. Let $0 \rightarrow \mathbf{g} \rightarrow \mathbf{h} \rightarrow \mathbf{f} \rightarrow 0$ be a short exact sequence in $\mathcal{G}$. Then this is an almost split sequence in $\mathcal{G}$ if and only if the sequence obtained by applying the cokernel functor $0 \rightarrow \mathbf{C o k}(\mathbf{g}) \rightarrow \operatorname{Cok}(\mathbf{h}) \rightarrow \operatorname{Cok}(\mathbf{f}) \rightarrow 0$ is an almost split sequence in $\mathcal{E}$.

Proof. By using the diagram chasing, one can easily show that $\mathbf{h} \rightarrow \mathbf{f}$ is right almost split in $\mathcal{G}$ if and only if $\operatorname{Cok}(\mathbf{h}) \rightarrow \operatorname{Cok}(\mathbf{f})$ is right almost split in $\mathcal{E}$. So Lemma 3.8.(i) completes the proof.

The result below describes the Auslander-Reiten translation in $\mathcal{E}$ within $\bmod R$.

Theorem 5.11. Let $(B \stackrel{\mathrm{g}}{\rightarrow} C)$ be an indecomposable non-projective object in $\mathcal{E}$ which is locally projective on the punctured spectrum of $R$. Then there is an almost split sequence in $\mathcal{E}$ ending at $\mathbf{g}$ and, in particular,

$$
\tau_{\mathcal{E}}(\mathbf{g}) \cong \operatorname{Cok}\left(\tau_{\mathcal{G}}(\operatorname{Ker}(\mathbf{g}))\right) \cong \operatorname{Cok}\left(\operatorname{red}\left(\mathcal{G}-\operatorname{cov} \tau_{R} \mathbf{g}\right)\right)
$$

Proof. Since $B \stackrel{\mathbf{g}}{\rightarrow} C$ is indecomposable, obviously the same is true for $\operatorname{Ker}(\mathbf{g})=$ $(\operatorname{Ker}(\mathbf{g}) \rightarrow B)$. In addition, the hypothesis imposed on $(B \stackrel{\mathbf{g}}{\rightarrow} C)$, induces that $\operatorname{Ker}(\mathbf{g})$ as an object of $\mathcal{G}$, is indecomposable non-projective and locally projective on the punctured spectrum of $R$. Therefore, Proposition 5.10 combining with Theorem 5.9 yields the desired result.

Proposition 5.12. Let $0 \rightarrow A \stackrel{\mathbf{f}}{\rightarrow} B \stackrel{\mathrm{g}}{\rightarrow} C \rightarrow 0$ be an almost split sequence in $\mathrm{G}(R)$.

(i) The almost split sequence in $\mathcal{G}$ and $\mathcal{H}$ ending at $(0 \rightarrow C)$ has the form

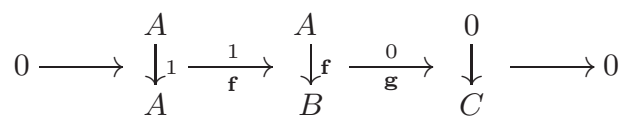


(ii) Let $\mathbf{e}: A \rightarrow P$ be a projective envelope of $A$. Then the almost split sequence in $\mathcal{G}$ ending at $(C \stackrel{1}{\rightarrow} C)$ has the form

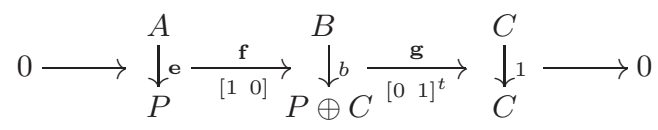

where $b$ is the map $[\mathbf{u} \mathbf{g}]$ with $\mathbf{u}: B \rightarrow P$ is an extension of the map $\mathbf{e}$.

(iii) The almost split sequence in $\mathcal{E}$ and $\mathcal{H}$ ending at $(C \stackrel{1}{\rightarrow} C)$ has the form

$$
0 \longrightarrow{\underset{0}{\downarrow}}_{0}^{A} \stackrel{\mathrm{f}}{\longrightarrow} \underset{C}{\stackrel{\downarrow}{\downarrow} \mathrm{g}} \stackrel{\mathrm{g}}{\longrightarrow} \underset{C}{\downarrow_{1}} \longrightarrow 0
$$

(iv) The almost split sequence in $\mathcal{E}$ ending at $(C \rightarrow 0)$ has the form

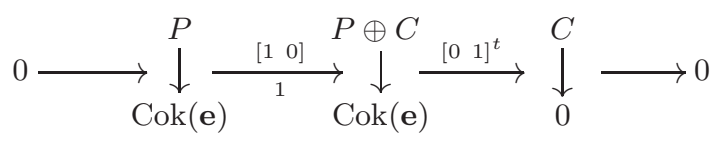

Proof. (i). It is easily verified that $C$ being non-projective, locally projective on the punctured spectrum of $R$ with local endomorphism ring, induces that the same is true for $(0 \rightarrow C)$ as an object in $\mathcal{H}$ (and also $\mathcal{G}$ ). So, because of Remark 5.2, there is an almost split sequence in $\mathcal{H}$ ending at $(0 \rightarrow C)$ and, in view of Corollary 3.9 in conjunction with Corollary $5.3, \tau_{\mathcal{H}}(0 \rightarrow C)$ has the form $(A \stackrel{1}{\rightarrow} A)$. Consequently, it is also an almost split sequence in $\mathcal{G}$, since $\mathcal{G}$ is resolving. That is, $\tau_{\mathcal{G}}(0 \rightarrow C)=\tau_{\mathcal{H}}(0 \rightarrow C)=(A \stackrel{1}{\rightarrow} A)$, as required.

(ii). Analogues to part $(i)$, there is an almost split sequence in $\mathcal{H}$ ending at $(C \stackrel{1}{\rightarrow} C)$. Making use of Corollary 3.9 again, we obtain that $\tau_{\mathcal{H}}(C \stackrel{1}{\rightarrow} C)=(A \rightarrow 0)$. Hence Theorem 5.9 combining with Corollary 5.8, gives the desired result.

(iii) and (iv). The results follow by applying the cokernel functor to almost split sequences appearing in the proof of parts $(i)$ and (ii), thanks to Proposition 5.10. The proof then is completed.

Remark 5.13. Let $A \in \mathrm{G}(R)$ be arbitrary. Then, by virtue of 2.5 , the isomorphism $\tau_{R}^{-1} A \cong\left(\tau_{R} A^{\prime}\right)^{\prime}$ holds true. In addition, for objects $\mathbf{f} \in \mathcal{G}, \mathbf{g} \in \mathcal{E}$, one has the isomorphisms; $\tau_{\mathcal{G}}^{-1}(\mathbf{f}) \cong\left(\tau_{\mathcal{E}}\left(\mathbf{f}^{\prime}\right)\right)^{\prime}, \tau_{\mathcal{E}}^{-1}(\mathbf{g}) \cong\left(\tau_{\mathcal{G}}\left(\mathbf{g}^{\prime}\right)\right)^{\prime}$, and $\tau_{\mathcal{H}}^{-1}(\mathbf{g}) \cong\left(\tau_{\mathcal{H}}\left(\mathbf{g}^{\prime}\right)\right)^{\prime}$.

We should stress again that, as we have pointed out in $2.5, \mathcal{G}$ and $\mathcal{E}$ are Frobenius categories, i.e., $\operatorname{proj} \mathcal{G}=\operatorname{inj} \mathcal{G}$ and $\operatorname{proj} \mathcal{E}=\operatorname{inj} \mathcal{E}$.

Similar to Theorems 5.9 and 5.11, one may deduce that for given indecomposable non-projective objects $\mathbf{f}$ and $\mathbf{g}$ in $\mathcal{G}$ and $\mathcal{E}$, respectively, there are almost split sequences starting at $\mathbf{f}$ and $\mathbf{g}$. In particular, we have the following.

Theorem 5.14. Let $\mathbf{f}$ and $\mathbf{g}$ be indecomposable non-projective objects in $\mathcal{G}$ and $\mathcal{E}$, respectively, which are locally projective on the punctured spectrum of $R$. Then,

(i) $\tau_{\mathcal{H}}^{-1}(\mathbf{g}) \cong \operatorname{Ker}\left(\operatorname{red}\left(\mathcal{E}-\right.\right.$ env $\left.\left.\tau_{R}^{-1} \mathbf{g}\right)\right) \cong \operatorname{red}\left(\mathcal{G}\right.$-cov $\left.\tau_{R}^{-1} \operatorname{Ker}(\mathbf{g})\right)$.

(ii) $\tau_{\mathcal{E}}^{-1}(\mathbf{g}) \cong \operatorname{red}\left(\mathcal{E}\right.$-env $\left.\tau_{R}^{-1} \operatorname{Ker}(\mathbf{g})\right)$.

(iii) $\tau_{\mathcal{G}}^{-1}(\mathbf{f}) \cong \operatorname{Ker}\left(\operatorname{red}\left(\mathcal{E}-\right.\right.$ env $\left.\left.\tau_{R}^{-1} \mathbf{f}\right)\right)$. 
Proof. (i). We have the following

$$
\begin{aligned}
\tau_{\mathcal{H}}^{-1}(\mathbf{g}) \cong\left(\tau_{\mathcal{H}}\left(\mathbf{g}^{\prime}\right)\right)^{\prime} & \cong\left(\mathbf{C o k}\left(\operatorname{red}\left(\mathcal{G}-\operatorname{cov} \tau_{R} \mathbf{g}^{\prime}\right)\right)^{\prime}\right. \\
& \cong \mathbf{K e r}\left(\operatorname{red}\left(\mathcal{E}-\operatorname{env}\left(\tau_{R} \mathbf{g}^{\prime}\right)^{\prime}\right)\right) \\
& \cong \mathbf{K e r}\left(\operatorname{red}\left(\mathcal{E}-\text { env } \tau_{R}^{-1} \mathbf{g}\right)\right)
\end{aligned}
$$

The first isomorphism follows from the previous remark and the second isomorphism holds true because of Corollary 5.3. The third isomorphism is valid, thanks to 2.11. Another use of the previous remark yields the last isomorphism. To achieve the second claimed isomorphism, similar to the preceding one, we have the following isomorphisms;

$$
\begin{aligned}
\tau_{\mathcal{H}}^{-1}(\mathbf{g}) \cong\left(\tau_{\mathcal{H}}\left(\mathbf{g}^{\prime}\right)\right)^{\prime} & \cong\left(\operatorname{red}\left(\mathcal{E}-\operatorname{env} \tau_{R} \mathbf{C o k}\left(\mathbf{g}^{\prime}\right)\right)\right)^{\prime} \\
& \cong \operatorname{red}\left(\mathcal{G}-\operatorname{cov} \tau_{R}\left(\mathbf{C o k}\left(\mathbf{g}^{\prime}\right)\right)^{\prime}\right) \\
& \cong \operatorname{red}\left(\mathcal{G}-\operatorname{cov}\left(\tau_{R}(\mathbf{K e r}(\mathbf{g}))^{\prime}\right)^{\prime}\right) \\
& \cong \operatorname{red}\left(\mathcal{G}-\operatorname{cov} \tau_{R}^{-1} \operatorname{Ker}(\mathbf{g})\right) .
\end{aligned}
$$

(ii). Consider the following isomorphisms;

$$
\begin{aligned}
\tau_{\mathcal{E}}^{-1}(\mathbf{g}) \cong\left(\tau_{\mathcal{G}}\left(\mathbf{g}^{\prime}\right)\right)^{\prime} & \cong\left(\operatorname{red}\left(\mathcal{G}-\operatorname{cov} \tau_{R}\left(\mathbf{C o k}\left(\mathbf{g}^{\prime}\right)\right)\right)\right)^{\prime} \\
& \cong \operatorname{red}\left(\mathcal{E}-\operatorname{env}\left(\tau_{R}(\mathbf{K e r}(\mathbf{g}))^{\prime}\right)^{\prime}\right) \\
& \cong \operatorname{red}\left(\mathcal{E}-\operatorname{env} \tau_{R}^{-1}(\mathbf{K e r}(\mathbf{g}))\right) .
\end{aligned}
$$

The first isomorphism follows from Remark 5.13, however the second one holds true, because of Theorem 5.9. The third isomorphism is true, as we have noted just above.

(iii). In order to obtain the last statement, we consider the following

$$
\begin{aligned}
\tau_{\mathcal{G}}^{-1}(\mathbf{f}) \cong\left(\tau_{\mathcal{E}}\left(\mathbf{f}^{\prime}\right)\right)^{\prime} & \cong\left(\mathbf{C o k}\left(\operatorname{red}\left(\mathcal{G}-\operatorname{cov} \tau_{R} \mathbf{f}^{\prime}\right)\right)\right)^{\prime} \\
& \cong \mathbf{K e r}\left(\operatorname{red}\left(\mathcal{E}-\operatorname{env}\left(\tau_{R} \mathbf{f}^{\prime}\right)^{\prime}\right)\right) \\
& \cong \operatorname{Ker}\left(\operatorname{red}\left(\mathcal{E}-\operatorname{env} \tau_{R}^{-1} \mathbf{f}\right)\right) .
\end{aligned}
$$

The validity of the first and last isomorphisms come from Remark 5.13. The second isomorphism is given in Theorem 5.11, however, the third isomorphism is obvious. So the proof is complete.

As an immediate consequence of Theorems 5.9 and 5.11 combining with the above theorem, we include the result below.

Corollary 5.15. Let $\mathbf{f}$ and $\mathbf{g}$ be arbitrary indecomposable non-projective objects in $\mathcal{G}$ and $\mathcal{E}$, respectively, which are locally projective on the punctured spectrum of $R$. Then $\mathbf{f}$ and $\mathbf{g}$ admit almost split sequences in $\mathcal{G}$ and $\mathcal{E}$, respectively.

Example 5.16. Let $A$ be a non-projective object of $\mathrm{G}(R)$ and $A \stackrel{\mathrm{f}}{\rightarrow} P$ be a projective envelope of $A$ with $\operatorname{End}_{\mathcal{H}}(\mathbf{f})$ is local. Assume, additionally, that $\mathbf{f}$ is a locally projective on the punctured spectrum of $R$. So, as we have seen earlier, there is an almost split sequence in $\mathcal{H}$ ending at $(A \rightarrow P)$. In particular, according to Corollary 5.3 together with Corollary 3.9, one has $\tau_{\mathcal{H}}(\mathbf{f})=\left(Q \rightarrow \tau_{R} L\right)$, whenever $L=\operatorname{Cok}(\mathbf{f})$ and $Q$ is a projective cover of $\tau_{R}(L)$. Moreover, since $A \in \mathrm{G}(R)$, we deduce that the rows of almost split sequence ending at $\mathbf{f}$ are split and so it has the form

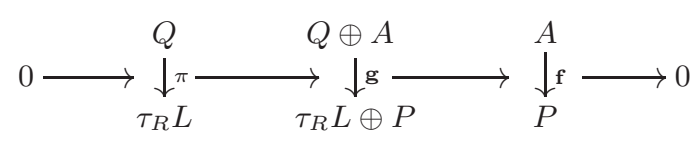

We would like to recognize the map g, explicitly. To do this, first we should remark that, in view of Proposition 3.8.(i), $\operatorname{End}_{\mathcal{H}}(P \rightarrow L)$ is local and hence, it can be easily 
seen that so is $\operatorname{End}_{R}(L)$. Also from the hypothesis made on $\mathbf{f}$, we may infer that the $R$-module $L$ is a non-projective which is locally projective on the punctured spectrum of $R$. Consequently, there is an almost split sequence ending at $L$ as follows;

$$
0 \rightarrow \tau_{R} L \rightarrow X \rightarrow L \rightarrow 0 .
$$

Hence, in view of the following commutative diagram in $\bmod R$;

we have that $\mathbf{g}=\left[\begin{array}{ll}\pi & 0 \\ \alpha & \mathbf{f}\end{array}\right]$.

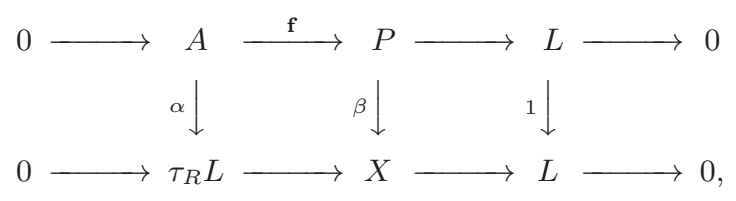

We close this paper by mentioning that the stable categories $\underline{\mathcal{G}}$ and $\underline{\mathcal{E}}$ admit AuslanderReiten triangles.

Remark 5.17. As we have mentioned in the introduction, the subcategories $\mathcal{G}$ and $\mathcal{E}$ are Frobenius. In fact, we have the following;

$$
\operatorname{proj} \mathcal{H}=\operatorname{proj} \mathcal{G}=\operatorname{inj} \mathcal{G} \quad \text { and } \quad \operatorname{inj} \mathcal{H}=\operatorname{inj} \mathcal{E}=\operatorname{proj} \mathcal{E} .
$$

So, the stable categories $\underline{\mathcal{G}}$ and $\underline{\mathcal{E}}$ are triangulated; see [16, Theorem 2.6]. Hence, almost split sequences in $\mathcal{G}$ and $\underline{\mathcal{E}}$ induce Auslander-Reiten triangles in $\underline{\mathcal{G}}$ and $\underline{\mathcal{E}}$, respectively. In particular, by making use of Theorems 5.9, 5.11 and 5.14, one may determine the Auslander-Reiten triangles in the categories $\underline{\mathcal{G}}$ and $\underline{\mathcal{E}}$. More precisely, suppose that $\mathcal{X}$ is an object of $\underline{\mathcal{G}}(\operatorname{resp} . \underline{\mathcal{E}})$ with local endomorphism $\operatorname{ring} \operatorname{End}_{\mathcal{G}}(\mathcal{X})\left(\operatorname{resp} . \operatorname{End}_{\underline{\mathcal{E}}}(\mathcal{X})\right)$. Then, it is easy to verify that the Auslander-Reiten triangles in $\underline{\mathcal{G}}$ and $\underline{\mathcal{E}}$ are as follows;

$$
\begin{aligned}
\tau_{\mathcal{G}} \mathcal{X} \rightarrow \mathcal{Y} \rightarrow \mathcal{X} \rightsquigarrow \quad \text { and } & \mathcal{X} \rightarrow \mathcal{Y} \rightarrow \tau_{\mathcal{G}}^{-1} \mathcal{X} \rightsquigarrow \\
\tau_{\mathcal{E}} \mathcal{X} \rightarrow \mathcal{Y} \rightarrow \mathcal{X} \rightsquigarrow \quad \text { and } & \mathcal{X} \rightarrow \mathcal{Y} \rightarrow \tau_{\mathcal{E}}^{-1} \mathcal{X} \rightsquigarrow .
\end{aligned}
$$

\section{REFERENCES}

[1] J. Asadollahi And R. Hafezi, On the dimensions of path algebras, Math. Res. Lett. 21 (2014), no. $1,19-31$.

[2] M. Auslander, Functors and morphisms determined by objects, Proc. Conf. on representation theory, pp. 1-244 (Philadelphia 1967). New York: Dekker 1978.

[3] M. Auslander, Comments on the functor Ext, Topology 8 (1969), 151-161.

[4] M. Auslander, Isolated singularities and the existence of almost split sequences, Proc. ICRA IV, Lecture Notes in Mathematics, vol. 1178, Springer-Verlag, New York-Berlin,1986, pp. 194-241.

[5] M. Auslander and I. Reiten, Representation theory of artin algebras III, Comm. Algebra 3 (1975), 239-294.

[6] M. Auslander and I. Reiten, Almost split sequences for Cohen-Macaulay modules, Math. Ann. 227 (1987), 345-349.

[7] M. Auslander, I. Reiten and S. O. Smal $\emptyset$, Representation theory of artin algebras. Cambridge University Press. (1995).

[8] A. Beligiannis, Relative homological algebra and purity in triangulated categories, J. Algebra 227 (2000), 268-361.

[9] G. Birkhoff, Subgroups of abelian groups, Proc. Lond. Math. Soc. (2)38 (1934), 385-401.

[10] X. W. Chen, An Auslander type result for Gorenstein projective modules, Adv. in Math. 218 (2008), 2043-2050.

[11] C. W. Curtis And I. Reiner, Methods of Representation Theory, Vol. 1, WileyInterscience, 1981. 
[12] E. Enochs and S. Estrada, Projective representations of quivers, Comm. Algebra 33 (2005), $3467-3478$

[13] E. Enochs, S. Estrada and J. R. Garci'a Rozas, Injective representations of infinite quivers. Applications, Canad. J. Math. 61 (2009), no. 2, 315-335.

[14] E. Enochs And I. Herzog, A homotopy of quiver morphisms with applications to representations, Canad. J. Math. 51 (2) (1999), 294-308.

[15] H. Eshraghi, R. Hafezi and Sh. Salarian, Total acyclicty for complexes of representations of quivers, Comm. Algebra 41 (2013), 4425-4441.

[16] D. HAPpel, Triangulated categories in the representation theory of finite dimensional algebra, Cambridge Univ. Press. 1988.

[17] B. Keller, Chain complexes and stable categories, Manuscripta Math. 67 (1990), 379-417.

[18] S. Liu, P. NG and C. Paquette, Almost split sequences and approximations, Algebr. Represent. Theor. 16 (2013) 1809-1827.

[19] A. Martsinkovsky and J. R. Strooker, Linkage of modules, J. Algebra 271 (2004), 587-626.

[20] I. Reiner, Maximal orders, London Mathematical Society Monographs, No. 5, Academic Press [A subsidiary of Harcourt Brace Jovanovich, Publishers], London-New York, 1975.

[21] C. M. Ringel And M. Schmidmeier, The Auslander-Reiten translation in submodule category, Trans. Amer. Math. Soc. 360 (2008), 691-716.

[22] W. Rump, The category of lattices over a lattice-finite ring, Algebras and Repr. Theory 8 (2005), 323-345.

[23] D. Simson, Linear representation types of the category of subprojective representations of a finite poset over $K[t] /\left(t^{m}\right)$ and a solution of a Birkhoff type problem, J. Algebra 311 (2007), 1-30.

[24] D. Simson And M. Wojewodzki, An algorithmic solution of a Birkhoff type problem, Fund. Inform. 83 (2008), 389-410.

[25] C. Peskin And L. SzPiro, Liaison des variétés algébriqeus. I, Invent. Math. 26 (1974), 271-302.

Department of Mathematics, Gonbade-Kavous University, Postal Code:4971799151, Gonbade KAVOUS, IRAN

E-mail address: n.bahlekeh@gmail.com

Department of Mathematics, University of Isfahan, P.O.Box: 81746-73441, Isfahan, Iran

E-mail address: amfallah@sci.ui.ac.ir

Department of Mathematics, University of Isfahan, P.O.Box: 81746-73441, Isfahan, Iran and, School of Mathematics, Institute for Research in Fundamental Science (IPM), P.O.BOX: 19395-5746, TEHRAN, IRAN

E-mail address: Salarian@ipm.ir 